\section{Butterflies of Kerala Agricultural UNIVERSITY (KAU) CAMPUS, THRISSUR, KERALA, INDIA}

\section{K.S. Aneesh ${ }^{1}$, C.K. Adarsh ${ }^{2} \&$ P.O. Nameer ${ }^{3}$}

${ }^{1}$ Assistant Professor, Department of Forestry and Wood Technology, Sir Syed College, Taliparamba, Kannur, Kerala 670142, India

${ }^{2}$ BSc final year (Forestry), ${ }^{3}$ Associate Professor and Head,

Department of Wildlife Sciences, College of Forestry, Kerala Agricultural

University, Thrissur, Kerala 680656, India

${ }^{1}$ aneeshkscof@gmail.com, ${ }^{2}$ adarshckcof09@gmail.com,

${ }^{3}$ nameerpo@gmail.com (corresponding author)

Butterflies are sensitive biota, which get severely affected by environmental variations and changes in forest structure (Pollard 1991). They form an important part of the food chain of birds, reptiles, amphibians, spiders and predatory insects. They also respond to disturbances and changes in the quality of habitat, and are thus a good indicator species to evaluate changes in habitat and landscape structure variations (Kremen 1992; Kocher \& Williams 2000). Butterflies and their caterpillars are dependent on specific host plants for food, thus the diversity of butterflies indirectly reflects overall plant diversity especially that of shrubs and herbs in the given area (Padhye et al. 2006). Most of them are strictly seasonal and prefer only particular set of habitats (Kunte 1997).

India has around 1,501 species of butterflies, out of which 334 species are reported from the Western Ghats and 37 species are endemic to the Western Ghats (Evans 1932; Kunte 2000). Of the 334 species of butterflies of Western Ghats, 316 species have been reported from Kerala (Palot et al. 2012).
Very little documentation has been done on butterfly fauna in Kerala. Some of the earlier documentation on butterfly fauna from Kerala and adjacent areas include Mathew \& Rahamathulla (1993), who had reported 100 species of butterflies from Silent

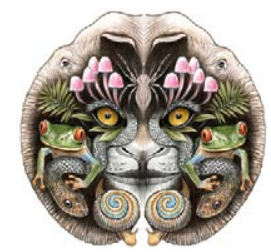

ISSN

Online 0974-7907 Print 0974-7893

\section{OPEN ACCESS} Valley National Park, Sudheendrakumar et al. (2000), who reported 124 species of butterflies from Parambikulam Wildlife Sanctuary, Arun (2003), reported 75 species from Siruvani Reserved Forests, Ambrose \& Raj (2005) reported 24 species from Kalakkad-Mundanthurai Tiger reserve, Eswaran \& Pramod (2005) reported 75 species from Anaikatty near Coimbatore, Prasad et al. (2010) recorded 52 species from Kerala University campus, Thiruvananthapuram, while Toms et al. (2010) reported 109 species from Mahatma Gandhi University campus, Kottayam. In the present study, an attempt has been made to document the richness of butterflies in Kerala Agricultural University (KAU) campus and the findings are presented in this paper.

Study area: The Kerala Agricultural University (KAU) main campus is located at Vellanikkara, Thrissur District, Kerala (Fig. 1). The area lies between $10^{\circ} 32^{\prime}-10^{\circ} 33^{\prime} \mathrm{N}$ and $76^{\circ} 16^{\prime}-76^{\circ} 17^{\prime} \mathrm{E}$ and is located very close to the PeechiVazhani Wildlife Sanctuary, Western Ghats, the aerial distance of which is not more than $5 \mathrm{~km}$. The campus has a total area of 391.44ha. and the major habitats include garden lands, botanical garden, plantations of rubber, coconut, plantain, cocoa and orchards of mango, jackfruit, sapota and guava. KAU campus enjoys a moderate climate. The ten-year mean minimum

DOI: http://dx.doi.org/10.11609/JoTT.o2870.4422-40 | ZooBank: urn:Isid:zoobank.org:pub:07D10F3A-D768-4C03-B4A0-54A16C30E2F1

Citation: Aneesh, K.S., C.K. Adarsh \& P.O. Nameer (2013). Butterflies of Kerala Agricultural University (KAU) campus, Thrissur, Kerala, India. Journal of Threatened Taxa 5(9): 4422-4440; http://dx.doi.org/10.11609/JoTT.o2870.4422-40

Copyright: ( ) Aneesh et al. 2013. Creative Commons Attribution 3.0 Unported License. JoTT allows unrestricted use of this article in any medium, reproduction and distribution by providing adequate credit to the authors and the source of publication.

Funding: Kerala Agricultural University.

Competing Interest: None.

Acknowledgements: The authors thank the two anonymous reviewers and the subject editor for their critical comments which greatly improved the manuscript. We thank Jobin K. Mathew, R. Arunraj, K. Haseena, K. Anu Sagaran, Y.N. Annie Jasmine for the support in the field. Mr. V.S. Sreehari for helping us in the preparation of the map. We also thank the Dean, College of Forestry, Kerala Agricultural University for encouragement and support. 


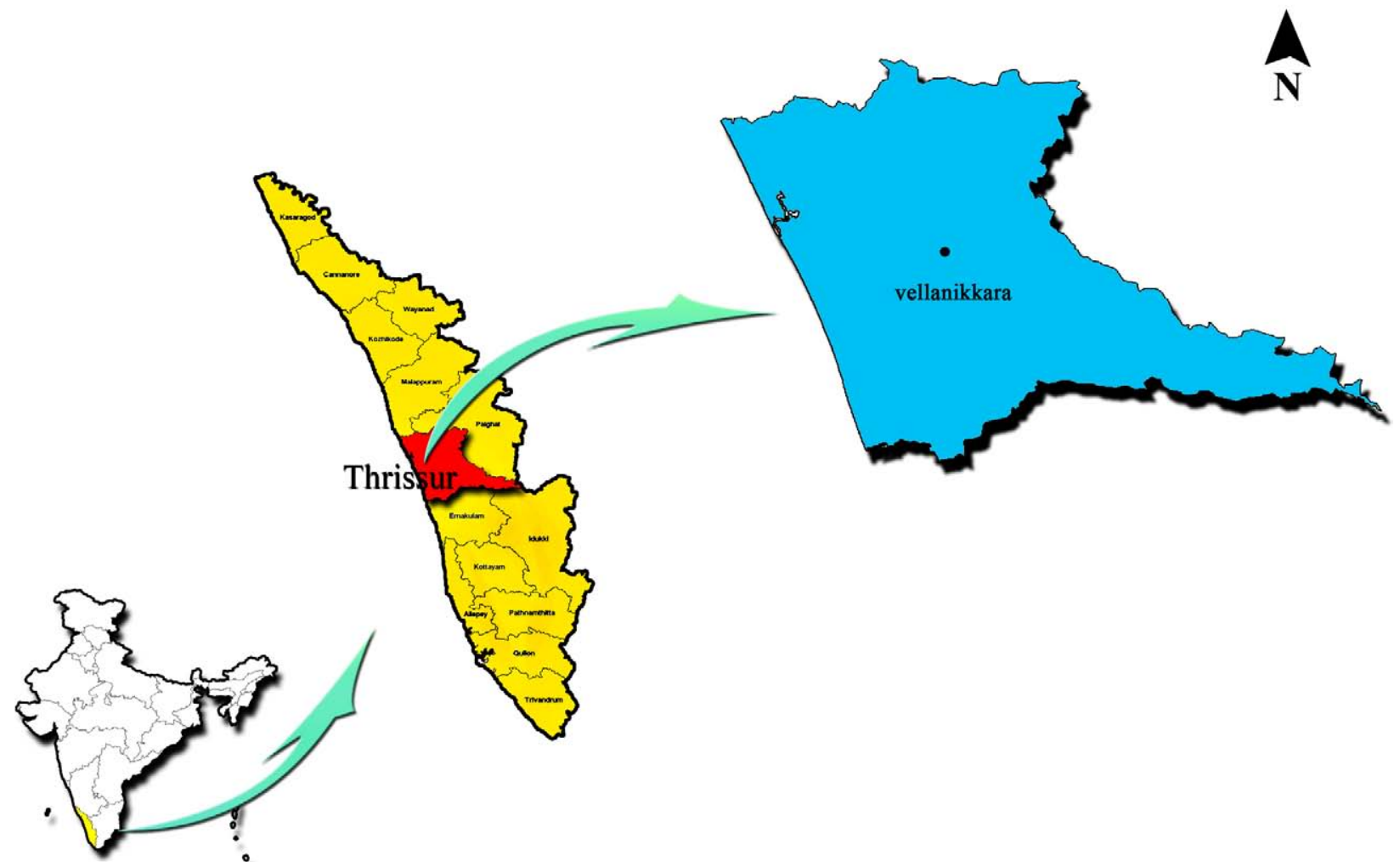

Figure 1. Location map of Kerala Agricultural University campus, Vellanikkara, Thrissur

temperature is $23.3^{\circ} \mathrm{C}$ and ten-year mean maximum of $31.8^{\circ} \mathrm{C}$. The area receives south-west and north-east monsoons, the greater portion of the rainfall, however is received from the south-west monsoon between June and September. The mean annual rainfall is $2763 \mathrm{~mm}$. The mean number of rainy days per year is 110 days (KAU weather station, 2010).

Methods: The butterfly fauna of the campus was surveyed from November 2009 to December 2011. The whole campus was divided into two broad habitats such as plantations (rubber, cocoa, banana and fruit orchards) with an area of $89.65 \mathrm{ha}$ and natural habitats having an area of 301.79ha. These two habitats were surveyed on foot at least once a week. The butterfly species were also photo-documented during the study period. Species identity was confirmed with the help of the field guides by Kunte (2000) and Kehimkar (2008), taxonomy and nomenclature have been updated after Kunte et al. (2011). Butterflies observed were categorized into five groups based on their abundance during the period of study. Accordingly, those species observed $80-100 \%$ of the survey days were categorized as very common (VC), 60-80\% as common (C), 40-60\% as occasional (O), 20$40 \%$ as rare (R) and below $20 \%$ as very rare (VR).
Results and Discussion: A total of 139 species of butterflies belonging to six families were identified from the KAU campus, including four species that are endemic to the Western Ghats and nine species protected under various schedules of the Indian Wildlife (Protection) Act, 1972 (Table 1).

The family-wise distribution of butterflies of KAU campus is given in Fig. 2. Family Nymphalidae (brushfooted butterflies) dominated the butterfly fauna of KAU campus with 44 species followed by Lycaenidae (blues) 35 species, Hesperiidae (skippers) 34 species, Pieridae (whites and yellows) 13 species, Papilionidae (swallowtails) 12 species, and Riodinidae (judies and punches) with a single species.

Among the two broad habitat types at KAU, species richness was found to be more in natural areas with 124 species followed by plantations (98) (Fig. 3). Habitat preferences of butterfly families (Fig. 4) also show that the natural habitats have more number of species when compared to the man-modified habitats.

This observation is quite significant and it emphasizes the importance of University campuses in the conservation of biological diversity of a region. 


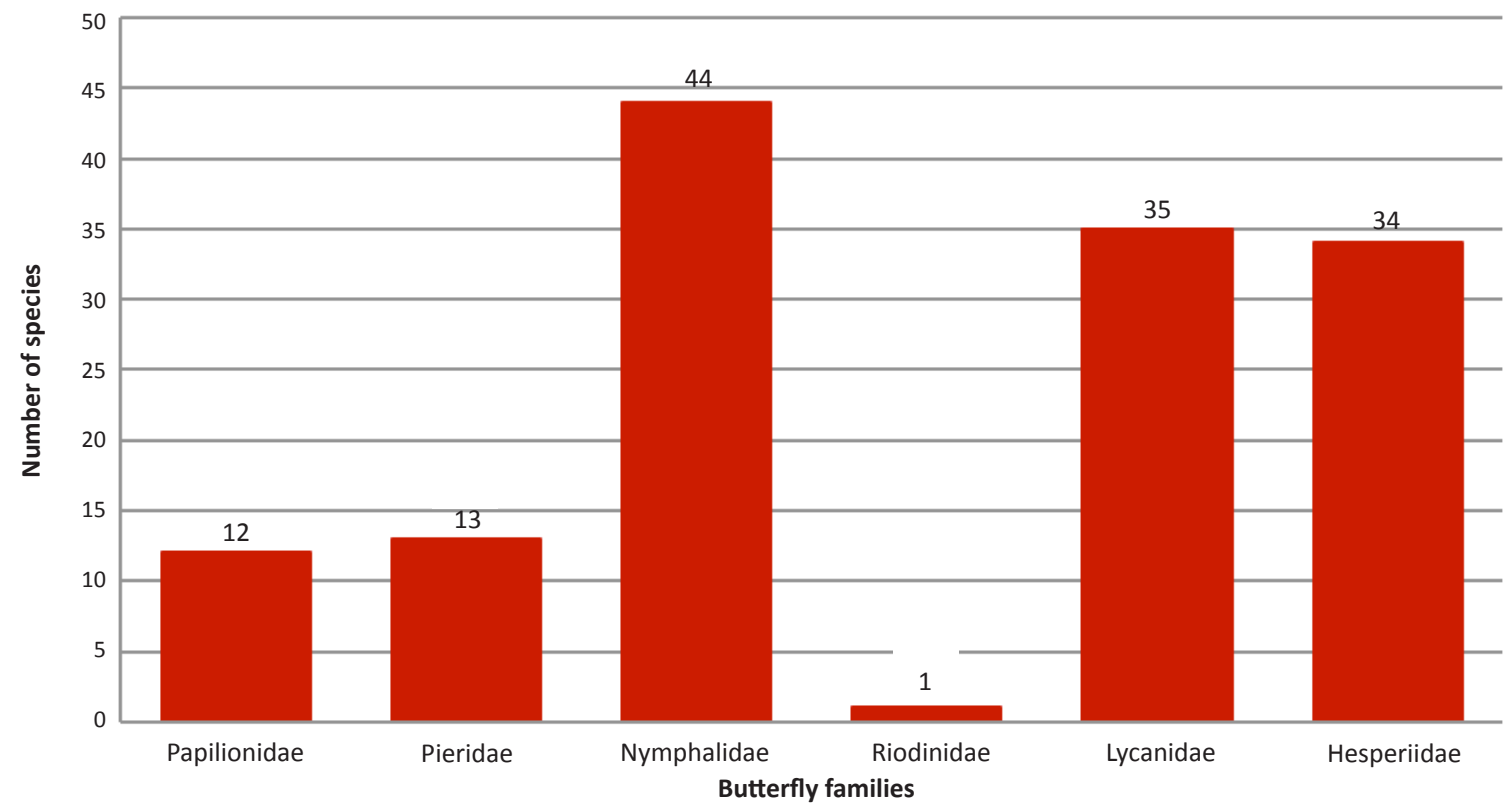

Figure 2. Family wise distribution of butterfly species in Kerala Agricultural University campus

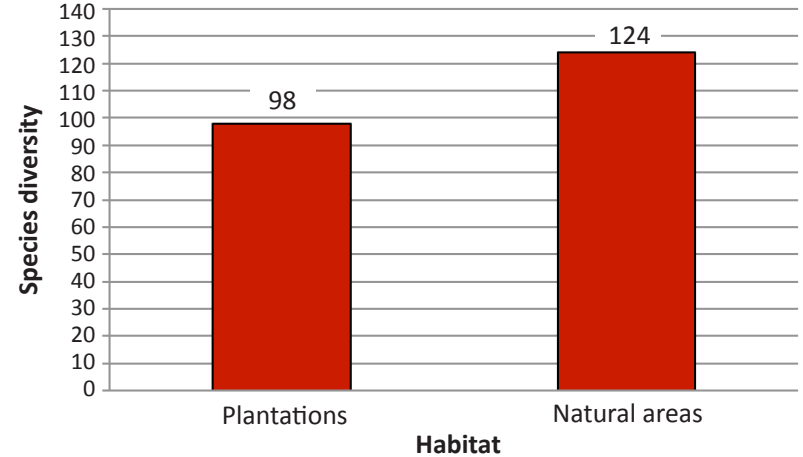

Figure 3. Butterfly species in the different habitats at Kerala Agricultural University campus

\section{REFERENCES}

Ambrose, D.P. \& D.S. Raj (2005). Butterflies of Kalakad-Mundanthurai Tiger Reserve, Tamil Nadu. Zoo's Print Journal 20(12): 2100-2107; http://dx.doi.org/10.11609/JoTT.ZPJ.1312.2100-7

Arun, P. R. (2003). Butterflies of Siruvani forests of Western Ghats with notes on their seasonality. Zoo's Print Journal 18(2):1003-1006; http://dx.doi.org/10.11609/JoTT.ZPJ.18.2.1003-6

Eswaran, R. \& P. Pramod (2005). Structure of butterfly community of Anaikatty Hills, Western Ghats. Zoo's Print Journal 20(8): 1939-1942; http://dx.doi.org/10.11609/JoTT.ZPJ.1330.1939-42

Evans, W.H. (1932). The Identification of Indian Butterflies. $2^{\text {nd }}$ Edition. Bombay Natural History Society, Mumbai, India, 464pp+32pl.

Kehimkar, I. (2008). The Book of Indian Butterflies. Bombay Natural History Society, 497pp.

Kocher, S.D. \& E.H. Williams (2000). The diversity and abundance of North American butterflies vary with habitat disturbances and geography. Journal of Biogeography 27: 785-794
Kremen, C. (1992). Assessing the Indicator Properties of Species Assemblages for Natural Areas Monitoring. Ecological Applications 2: 203-217.

Kunte, K. (1997). Seasonal patterns in butterfly abundance and species diversity in four tropical habitats in northern Western Ghats. Journal of Biosciences 22: 593-603.

Kunte, K. (2000). Butterflies of Peninsular India. Universities Press (Hyderabad) and Indian Academy of Sciences (Bengaluru), 270pp.

Kunte, K., S. Kalesh \& U. Kodandaramaiah (eds.). (2011). Butterflies of India. v. 1.03. Indian Foundation for Butterflies, Bengaluru.<http:// ifoundbutterflies.org/>, Accessed 2012.

Mathew, G. \& V.K. Rahamathulla (1993). Studies on the butterflies of Silent Valley National Park. Entomon 18(3): 185-192.

Palot, M.J., V.C. Balakrishnan \& S. Kalesh (2012). An updated checklist of butterflies of Kerala, with their Malayalam names. Malabar Trogon 9(3): 22-29.

Pollard, E. (1991). Monitoring butterfly numbers, pp. 87-111. In: Goldsmith, F.B (ed.). Monitoring for Conservation and Ecology. Chapman and Hall, London, 275pp.

Prasad, G., P.V. Prathibakumari \& A.M. Lizby (2010). Butterflies of Kerala University Campus, Thiruvananthapuram, Kerala. $3^{\text {rd }}$ Asian Lepidoptera Conservation Symposium and Training Programme, 25-29 October 2010, Coimbatore, India.

Sudheendrakumar, V. V., C. F. Binoy, P.V. Suresh \& G. Mathew (2000). Habitat association of butterflies in the Parambikulam Wildlife Sanctuary, Kerala, India. Journal of the Bombay Natural History Society 97(2): 193-201.

Toms, A., S.P. Narayanan, Babu, V. Padmakumar, B. Arun, N.D. Jaisen, J. Paul, M. deepa, K. Jisha, K.K. Jayasooryan, J. Ranjini, C. Rathy, P.N. Sreejith, G. Christopher \& A.P. Thomas (2010). Butterfly fauna of the Mahatma Gandhi University campus, Kerala and the strategies adopted for its conservation. 3rd Asian Lepidoptera Conservation Symposium and Training Programme, 25-29 October 2010, Coimbatore, India. 


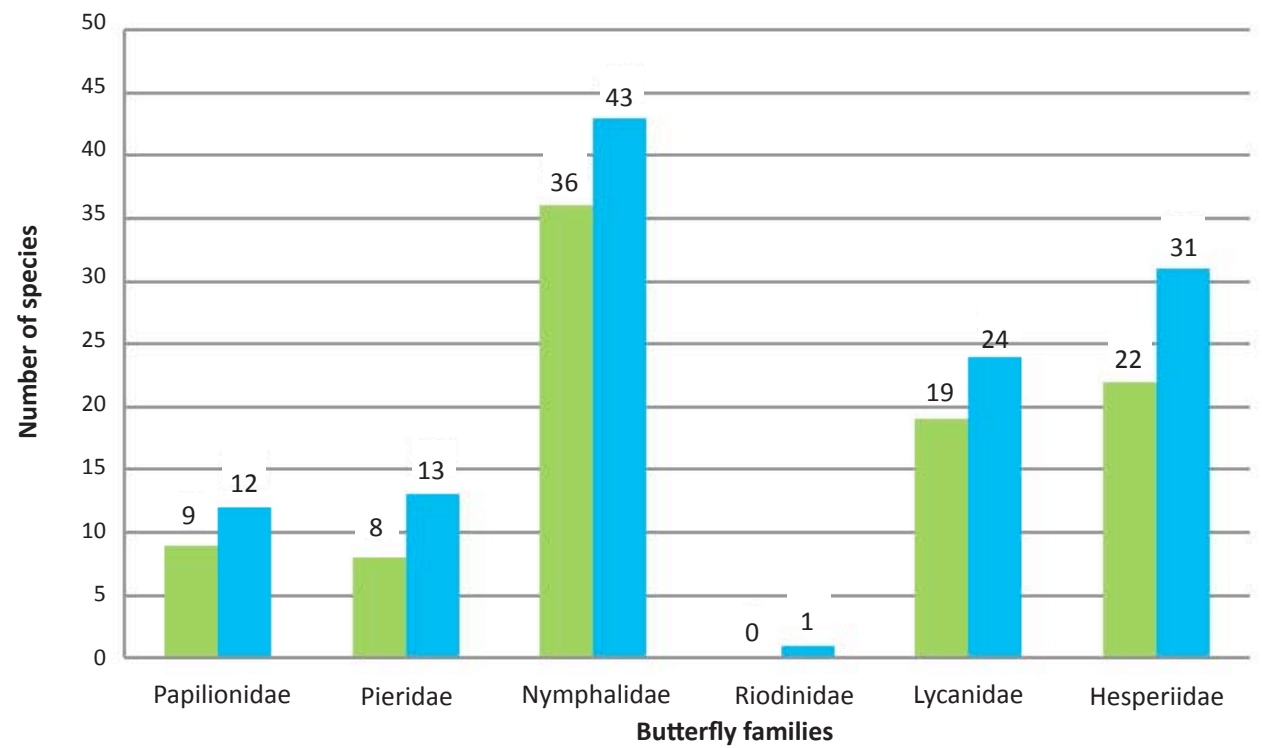

Plantations

Natural trees

Figure 4. Habitat preference of the butterfly families at Kerala Agricultural University campus

Table 1. Checklist of butterflies of Kerala Agricultural University campus, Thrissur, Kerala

\begin{tabular}{|c|c|c|c|c|c|c|}
\hline & Scientific name & Common name & Family & Subfamily & $\begin{array}{l}\text { Status in } \\
\text { IWPA } 1972\end{array}$ & $\begin{array}{c}\text { Relative } \\
\text { Abundance }\end{array}$ \\
\hline 1 & Troides minos** (Image 1 ) & Southern Birdwing & Papilionidae & Papilioninae & & C \\
\hline 2 & Pachliopta aristolochiae aristolochiae (Image 112) & Common Rose & Papilionidae & Papilioninae & & 0 \\
\hline 3 & Pachliopta hector (Image 2) & Crimson Rose & Papilionidae & Papilioninae & Schedule I & $\mathrm{O}$ \\
\hline 4 & Graphium sarpedon sarpedon (Image 3) & Common Bluebottle & Papilionidae & Papilioninae & & VR \\
\hline 5 & Graphium doson eleius (Image 4) & Common Jay & Papilionidae & Papilioninae & & $\mathrm{R}$ \\
\hline 6 & Graphium agamemnon menides (Image 5) & Tailed Jay & Papilionidae & Papilioninae & & C \\
\hline 7 & Papilio clytia clytia (Image 6) & Common Mime & Papilionidae & Papilioninae & & VR \\
\hline 8 & Papilio demoleus demoleus (Image 7) & Lime Butterfly & Papilionidae & Papilioninae & & C \\
\hline 9 & Papilio dravidarum** (Image 8) & Malabar Raven & Papilionidae & Papilioninae & & VR \\
\hline 10 & Papilio helenus daksha (Image 9) & Red Helen & Papilionidae & Papilioninae & & VR \\
\hline 11 & Papilio polytes romulus (Image 11) & Common Mormon & Papilionidae & Papilioninae & & C \\
\hline 12 & Papilio polymnestor polymnestor (Image 10) & Blue Mormon & Papilionidae & Papilioninae & & 0 \\
\hline 13 & Catopsilia pomona pomona (Image 12) & Common Emigrant & Pieridae & Coliadinae & & vc \\
\hline 14 & Catopsilia pyranthe pyranthe & Mottled Emigrant & Pieridae & Coliadinae & & C \\
\hline 15 & Eurema hecabe hecabe (Image 13) & Common Grass Yellow & Pieridae & Coliadinae & & VC \\
\hline 16 & Eurema brigitta rubella (Image 14) & Small Grass yellow & Pieridae & Coliadinae & & 0 \\
\hline 17 & Eurema blanda silhetana (Image 15) & Three-spot Grass Yellow & Pieridae & Coliadinae & & 0 \\
\hline 18 & Delias eucharis (Image 16) & Common Jezebel & Pieridae & Pierinae & & 0 \\
\hline 19 & Prioneris sita (Image 113) & Painted Sawtooth & Pieridae & Pierinae & Schedule IV & VR \\
\hline 20 & Cepora nerissa phryne (Image 17) & Common Gull & Pieridae & Pierinae & & $\mathrm{R}$ \\
\hline 21 & Belenois aurota aurota (Image 18) & Pioneer & Pieridae & Pierinae & & 0 \\
\hline 22 & Leptosia nina nina (Image 19) & Psyche & Pieridae & Pierinae & & VC \\
\hline 23 & Appias lyncida latifasciata (Image 20) & Chocolate Albatross & Pieridae & Pierinae & Schedule II & $\mathrm{R}$ \\
\hline 24 & Hebemoia glaucippe australis (Image 114) & Great Orange Tip & Pieridae & Pierinae & & $\mathrm{O}$ \\
\hline
\end{tabular}




\begin{tabular}{|c|c|c|c|c|c|c|}
\hline & Scientific name & Common name & Family & Subfamily & $\begin{array}{l}\text { Status in } \\
\text { IWPA } 1972\end{array}$ & $\begin{array}{c}\text { Relative } \\
\text { Abundance }\end{array}$ \\
\hline 25 & Pareronia hippie hippia (Image 21) & Common Wanderer & Pieridae & Pierinae & & VR \\
\hline 26 & Melanitis leda leda (Image 22) & Common Evening Brown & Nymphalidae & Satyrinae & & VC \\
\hline 27 & Elymnias hypermnestra undularis (Image 23) & Common Palmfly & Nymphalidae & Satyrinae & & C \\
\hline 28 & Lethe europa eoropa (Image 24) & Bamboo Treebrown & Nymphalidae & Satyrinae & & $\mathrm{O}$ \\
\hline 29 & Lethe drypetis todara (Image 25) & Tamil Treebrown & Nymphalidae & Satyrinae & & $\mathrm{R}$ \\
\hline 30 & Mycalesis perseus tabitha (Image 26) & Common Bushbrown & Nymphalidae & Satyrinae & & VC \\
\hline 31 & Orsotriaena medus mandata (Image 27) & Nigger & Nymphalidae & Satyrinae & & C \\
\hline 32 & Ypthima huebneri (Image 28) & Common Four-ring & Nymphalidae & Satyrinae & & VC \\
\hline 33 & Ypthima baldus madrasa (Image 29) & Common Five-ring & Nymphalidae & Satyrinae & & 0 \\
\hline 34 & Charaxes athamas athamas (Image 115) & Common Nawab & Nymphalidae & Charaxinae & & $\mathrm{R}$ \\
\hline 35 & Charaxes bemardus & Tawny Rajah & Nymphalidae & Charaxinae & & VR \\
\hline 36 & Charaxes solon solon (Image 30) & Black Rajah & Nymphalidae & Charaxinae & & VR \\
\hline 37 & Acraea violae (Image 31) & Tawny Coster & Nymphalidae & Heliconiinae & & VC \\
\hline 38 & Vindula erota saloma & Cruiser & Nymphalidae & Heliconiinae & & $\mathrm{R}$ \\
\hline 39 & Cupha erymanthis maja (Image 32) & Rustic & Nymphalidae & Heliconiinae & & C \\
\hline 40 & Phalanta phalantha phalantha (Image 33) & Common Leopard & Nymphalidae & Heliconiinae & & 0 \\
\hline 41 & Phalanta alcippe mercea (Image 34) & Small Leopard & Nymphalidae & Heliconiinae & & $\mathrm{O}$ \\
\hline 42 & Cirrochroa thais thais (Image 35) & Tamil Yeoman & Nymphalidae & Heliconiinae & & $\mathrm{O}$ \\
\hline 43 & Neptis jumbah nalanda (Image 36) & Chestnut-Streaked Sailer & Nymphalidae & Limenitidinae & & 0 \\
\hline 44 & Neptis hylas varmona (Image 37) & Common Sailer & Nymphalidae & Limenitidinae & & VC \\
\hline 45 & Pantoporia hordonia hordonia (Image 38) & Common Lascar & Nymphalidae & Limenitidinae & & C \\
\hline 46 & Athyma ranga karwara (Image 39) & Blackvein Sergeant & Nymphalidae & Limenitidinae & Schedule II & $\mathrm{R}$ \\
\hline 47 & Limenitis procris (Image 40) & Commander & Nymphalidae & Limenitidinae & & $\mathrm{O}$ \\
\hline 48 & Parthenos sy/via virens (Image 41) & Clipper & Nymphalidae & Limenitidinae & Schedule II & 0 \\
\hline 49 & Tanaecia lepidea miyana (Image 42) & Grey Count & Nymphalidae & Limenitidinae & Schedule II & 0 \\
\hline 50 & Euthalia aconthea meridionalis (Image 43) & Common Baron & Nymphalidae & Limenitidinae & & 0 \\
\hline 51 & Ariadne ariadne indica (Image 44) & Angled Castor & Nymphalidae & Biblidinae & & VC \\
\hline 52 & Ariadne merione merione (Image 45) & Common Castor & Nymphalidae & Biblidinae & & VC \\
\hline 53 & Junonia hierta hierta (Image 46) & Yellow Pansy & Nymphalidae & Nymphalinae & & VR \\
\hline 54 & Junonia orithya swinhoei (Image 47) & Blue Pansy & Nymphalidae & Nymphalinae & & VR \\
\hline 55 & Junonia lemonias lemonias (Image 48) & Lemon Pansy & Nymphalidae & Nymphalinae & & C \\
\hline 56 & Junonia almanac almana (Image 49) & Peacock Pansy & Nymphalidae & Nymphalinae & & $\mathrm{C}$ \\
\hline 57 & Junonia atlites atlites (Image 50) & Grey Pansy & Nymphalidae & Nymphalinae & & VC \\
\hline 58 & Junonia iphita iphita (Image 51) & Chocolate Pansy & Nymphalidae & Nymphalinae & & VC \\
\hline 59 & Hypolimnas misippus (Image 52) & Danaid Eggfly & Nymphalidae & Nymphalinae & Schedule I & $\mathrm{C}$ \\
\hline 60 & Hypolimnas bolina jacintha (Image 53) & Great Eggfly & Nymphalidae & Nymphalinae & & C \\
\hline 61 & Kaniska canace viridis (Image 116) & Blue Admiral & Nymphalidae & Nymphalinae & & $\mathrm{R}$ \\
\hline 62 & Parantica aglea aglea (Image 117) & Glassy Blue Tiger & Nymphalidae & Danainae & & 0 \\
\hline 63 & Tirumala limniace exoticus (Image 54) & Blue Tiger & Nymphalidae & Danainae & & 0 \\
\hline 64 & Tirumala septentrionis dravidarum (Image 55) & Dark Blue Tiger & Nymphalidae & Danainae & & 0 \\
\hline 65 & Danaus chrysippus chrysippus (Image 56) & Plain Tiger & Nymphalidae & Danainae & & $\mathrm{C}$ \\
\hline 66 & Danaus genutia genutia (Image 57) & Striped Tiger & Nymphalidae & Danainae & & C \\
\hline 67 & Euploea core core (Image 58) & Common Crow & Nymphalidae & Danainae & & VC \\
\hline
\end{tabular}




\begin{tabular}{|c|c|c|c|c|c|c|}
\hline & Scientific name & Common name & Family & Subfamily & $\begin{array}{c}\text { Status in } \\
\text { IWPA } 1972\end{array}$ & $\begin{array}{c}\text { Relative } \\
\text { Abundance }\end{array}$ \\
\hline 68 & Euploea sylvester coreta & Double Branded Crow & Nymphalidae & Danainae & & 0 \\
\hline 69 & Euploea klugii kollari (Image 59) & Brown King Crow & Nymphalidae & Danainae & & $\mathrm{R}$ \\
\hline 70 & Abiserae cherius (Image 60) & Plum Judy & Riodinidae & Nemeobiinae & & $\mathrm{R}$ \\
\hline 71 & Spalgis epius epeus (Image 61) & Apefly & Lycaenidae & Miletinae & & $\mathrm{R}$ \\
\hline 72 & Castalius rosimon rosimon (Image 62) & Common Pierrot & Lycaenidae & Polyommatinae & & VC \\
\hline 73 & Caleta caleta decidia (Image 63) & Angled Pierrot & Lycaenidae & Polyommatinae & & $\mathrm{R}$ \\
\hline 74 & Discolampa ethion ethion (Image 64) & Banded Blue Pierrot & Lycaenidae & Polyommatinae & & C \\
\hline 75 & Chilades pandava pandava (Image 65) & Plains Cupid & Lycaenidae & Polyommatinae & & $\mathrm{O}$ \\
\hline 76 & Acytolepis puspa felderi (Image 66) & Common Hedge Blue & Lycaenidae & Polyommatinae & & C \\
\hline 77 & Neopithecops zalmora dharma (Image 67) & Quaker & Lycaenidae & Polyommatinae & & C \\
\hline 78 & Zizina otis indica (Image 68) & Lesser Grass Blue & Lycaenidae & Polyommatinae & & C \\
\hline 79 & Zizula hylax hylax (Image 69) & Tiny Grass Blue & Lycaenidae & Polyommatinae & & C \\
\hline 80 & Pseudozizeeria maha ossa (Image 70) & Pale Grass Blue & Lycaenidae & Polyommatinae & & $\mathrm{R}$ \\
\hline 81 & Zizeeria karsandra & Dark Grass Blue & Lycaenidae & Polyommatinae & & $\mathrm{R}$ \\
\hline 82 & Euchrysopsc nejus cnejus (Image 71) & Gram Blue & Lycaenidae & Polyommatinae & Schedule II & VC \\
\hline 83 & Freyeria putli (Image 72) & Grass Jewel & Lycaenidae & Polyommatinae & & C \\
\hline 84 & Lampides boeticus (Image 73) & Pea Blue & Lycaenidae & Polyommatinae & Schedule II & VR \\
\hline 85 & Jamides celeno celeno (Image 74) & Common Cerulean & Lycaenidae & Polyommatinae & & C \\
\hline 86 & Jamides bochus bochus & Dark Cerulean & Lycaenidae & Polyommatinae & & VR \\
\hline 87 & Jamides alecto eurysaces & Metallic Cerulean & Lycaenidae & Polyommatinae & & VR \\
\hline 88 & Prosotas nora airdates (Image 75) & Common Line-blue & Lycaenidae & Polyommatinae & & VR \\
\hline 89 & Talicada nyseus nyseus (Image 76) & Red Pierrot & Lycaenidae & Polyommatinae & & $\mathrm{R}$ \\
\hline 90 & Anthene lycaenina lycaenina (Image 77) & Pointed Ciliate blue & Lycaenidae & Polyommatinae & & $\mathrm{R}$ \\
\hline 91 & Azanusu baldus & Bright Babul Blue & Lycaenidae & Polyommatinae & & VR \\
\hline 92 & Chilades lajus lajus (Image 78) & Lime Blue & Lycaenidae & Polyommatinae & & C \\
\hline 93 & Leptotes plinius plinius (Image 118) & Zebra Blue & Lycaenidae & Polyommatinae & & C \\
\hline 94 & Arhopala pseudocentaurus (Image 79) & Western Centaur Oakblue & Lycaenidae & Theclinae & & $\mathrm{R}$ \\
\hline 95 & Surendra quercetorum biplagiata (Image 80) & Common Acacia Blue & Lycaenidae & Theclinae & & $\mathrm{R}$ \\
\hline 96 & Spinda sisvulcanus vulcanus (Image 81) & Common Silverline & Lycaenidae & Theclinae & & C \\
\hline 97 & Spindasis elima (Image 82) & Scarce Shot Silverline & Lycaenidae & Theclinae & & $\mathrm{R}$ \\
\hline 98 & Loxura atymnus atymnus (Image 83) & Yamfly & Lycaenidae & Theclinae & & $\mathrm{O}$ \\
\hline 99 & Rapala manea schistacea (Image 84) & Slate Flash & Lycaenidae & Theclinae & & $\mathrm{O}$ \\
\hline 100 & Cheritra freja butleri (Image 85) & Common Imperial & Lycaenidae & Theclinae & & VR \\
\hline 101 & Rathinda amor (Image 86) & Monkey Puzzle & Lycaenidae & Theclinae & & 0 \\
\hline 102 & Zesius chrysomallus (Image 119) & Red Spot & Lycaenidae & Theclinae & & $\mathrm{R}$ \\
\hline 103 & Virachola isocrates (Image 120) & Common Guava Blue & Lycaenidae & Theclinae & & C \\
\hline 104 & Rachana jalindra macanita (Image 121) & Banded Royal & Lycaenidae & Theclinae & & VR \\
\hline 105 & Curetis thetis (Image 122) & Indian Sunbeam & Lycaenidae & Curetinae & & $\mathrm{R}$ \\
\hline 106 & Hasora chromus chromus (Image 123) & Common Banded Awl & Hesperiidae & Coeliadinae & & $\mathrm{R}$ \\
\hline 107 & Hasora badra badra & Common Awl & Hesperiidae & Coeliadinae & & $\mathrm{R}$ \\
\hline 108 & Badamia exclamationis (Image 124) & Brown Awl & Hesperiidae & Coeliadinae & & $\mathrm{O}$ \\
\hline 109 & Aeromachus pygmaeus (Image 87) & Pygmy Scrub Hopper & Hesperiidae & Hesperiinae & & 0 \\
\hline 110 & Halpe homo & Indian Ace & Hesperiidae & Hesperiinae & & $R$ \\
\hline
\end{tabular}




\begin{tabular}{|c|c|c|c|c|c|c|}
\hline & Scientific name & Common name & Family & Subfamily & $\begin{array}{l}\text { Status in } \\
\text { IWPA } 1972\end{array}$ & $\begin{array}{l}\text { Relative } \\
\text { Abundance }\end{array}$ \\
\hline 111 & Thoressa honorei** & Madras Ace & Hesperiidae & Hesperiinae & & VR \\
\hline 112 & Thoressaa stigmata** (Image 88) & Unbranded Ace & Hesperiidae & Hesperiinae & & VR \\
\hline 113 & Halpe porus (Image 89) & Moore's Ace & Hesperiidae & Hesperiinae & & VR \\
\hline 114 & Ampittia dioscorides dioscorides (Image 90) & Bush Hopper & Hesperiidae & Hesperiinae & & VC \\
\hline 115 & lambrix salsala luteipalpis (Image 91) & Chestnut Bob & Hesperiidae & Hesperiinae & & VC \\
\hline 116 & Psolos fuligo subfasciatus (Image 92) & Coon & Hesperiidae & Hesperiinae & & C \\
\hline 117 & Notocrypta paralysos mangla (Image 93) & Common Banded Demon & Hesperiidae & Hesperiinae & & 0 \\
\hline 118 & Notocrypta curvifascia curvifascia (Image 125) & Restricted Demon & Hesperiidae & Hesperiinae & & 0 \\
\hline 119 & Udaspes folus (Image 94) & Grass Demon & Hesperiidae & Hesperiinae & & 0 \\
\hline 120 & Suastus gremius gremius (Image 95) & Indian Palm Bob & Hesperiidae & Hesperiinae & & c \\
\hline 121 & Gangara thyrsis thyrsis (Image 96) & Giant Red-eye & Hesperiidae & Hesperiinae & & 0 \\
\hline 122 & Matapa aria (Image 97) & Common Red-eye & Hesperiidae & Hesperiinae & & c \\
\hline 123 & Taractro ceramaevius (Image 98) & Common Grass Dart & Hesperiidae & Hesperiinae & & 0 \\
\hline 124 & Taractro ceraceramas nicevillei & Tamil Grass Dart & Hesperiidae & Hesperiinae & & $\mathrm{R}$ \\
\hline 125 & Telicota colon colon (Image 99) & Pale Palm Dart & Hesperiidae & Hesperiinae & & $\mathrm{O}$ \\
\hline 126 & Telicota ancilla (Image 100) & Dark Palm Dart & Hesperiidae & Hesperiinae & & 0 \\
\hline 127 & Borbo bevani (Image 101) & Bevan's Swift & Hesperiidae & Hesperiinae & & VR \\
\hline 128 & Borbo cinnara (Image 102) & Rice Swift & Hesperiidae & Hesperiinae & & VC \\
\hline 129 & Pelopidas mathias mathias (Image 103) & Small Branded Swift & Hesperiidae & Hesperiinae & & 0 \\
\hline 130 & Pelopidas conjunctanarooa & Conjoined Swift & Hesperiidae & Hesperiinae & & $\mathrm{O}$ \\
\hline 131 & Tagiades gana silvia (Image 104) & Suffused Snow Flat & Hesperiidae & Pyrginae & & $\mathrm{O}$ \\
\hline 132 & Tagiades litigiosa litigiosa (Image 105) & Water Snow Flat & Hesperiidae & Pyrginae & & 0 \\
\hline 133 & Gerosis bhagava bhagava (Image 106) & $\begin{array}{l}\text { Common Yellow-breasted } \\
\text { Flat }\end{array}$ & Hesperiidae & Pyrginae & & $\mathrm{R}$ \\
\hline 134 & Pseudocoladenia dan dan (Image 107) & Fulvous Pied Flat & Hesperiidae & Pyrginae & & $\mathrm{O}$ \\
\hline 135 & Coladenia indrani indra (Image 108) & Tricoloured Pied Flat & Hesperiidae & Pyrginae & & VR \\
\hline 136 & Sarangesa dasahara dasahara (Image 109) & Common Small Flat & Hesperiidae & Pyrginae & & C \\
\hline 137 & Celaenorrhinus leucocera & Common Spotted Flat & Hesperiidae & Pyrginae & & 0 \\
\hline 138 & $\begin{array}{l}\text { Odontoptilum ransonnetii potiphera (Image } \\
\text { 110) }\end{array}$ & Golden Angle & Hesperiidae & Pyrginae & & $\mathrm{R}$ \\
\hline 139 & Spialia galba (Image 111) & Indian Grizzled Skipper & Hesperiidae & Pyrginae & & C \\
\hline
\end{tabular}

** Endemic to Western Ghats; VC-Very Common; C-Common; O-Occasional; R-Rare; VR-Very Rare 


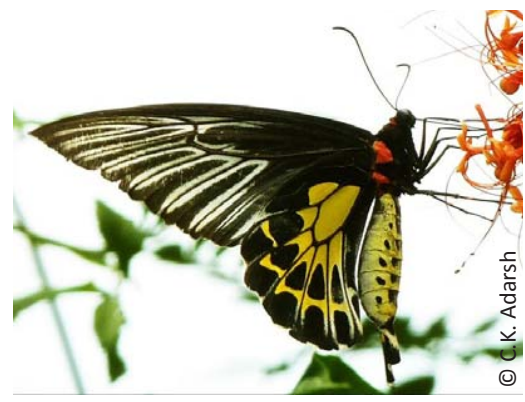

Image 1. Troides minos Southern Birdwing

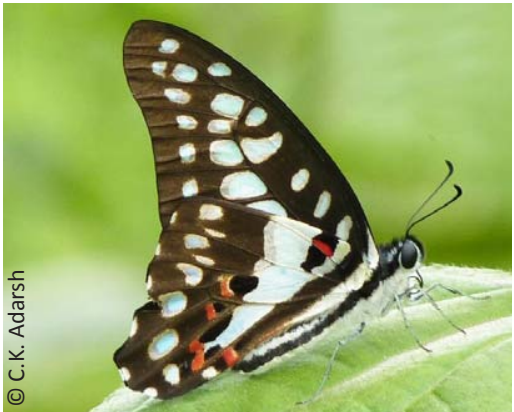

Image 4. Graphium doson Common Jay

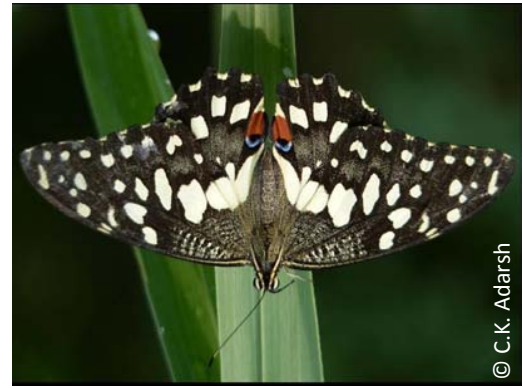

Image 7. Papilio demoleus Lime Butterfly

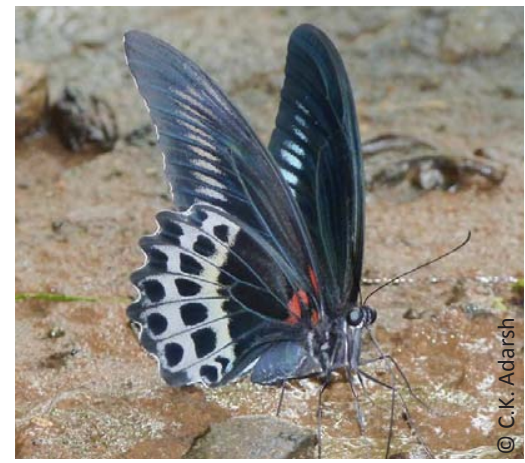

Image 10. Papilio polymnestor Blue Mormon

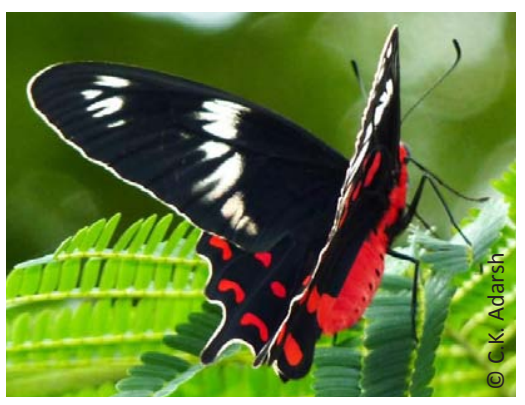

Image 2. Pachliopta hector Crimson Rose

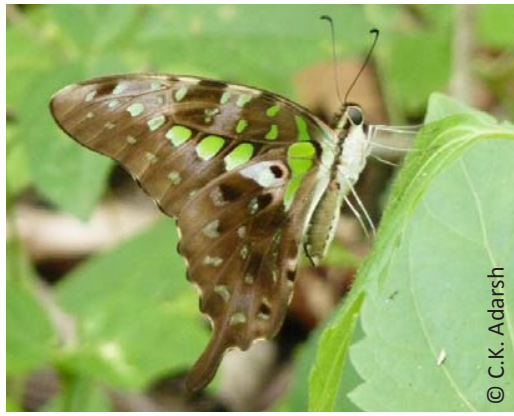

Image 5. Graphium agamemnon Tailed Jay

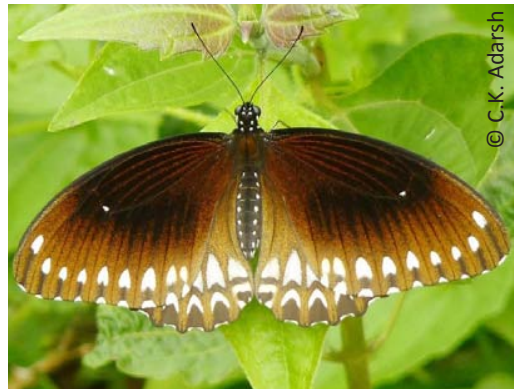

Image 8. Papilio dravidarum Malabar Raven

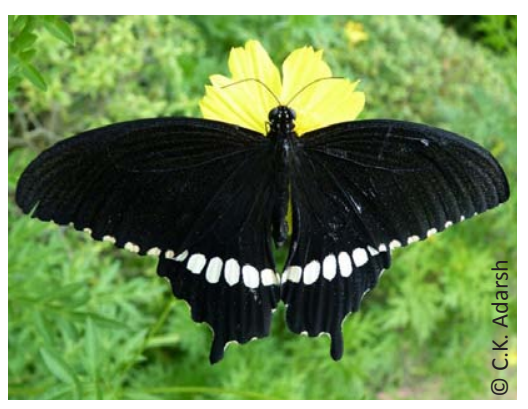

Image 11. Papilio polytes Common Mormon

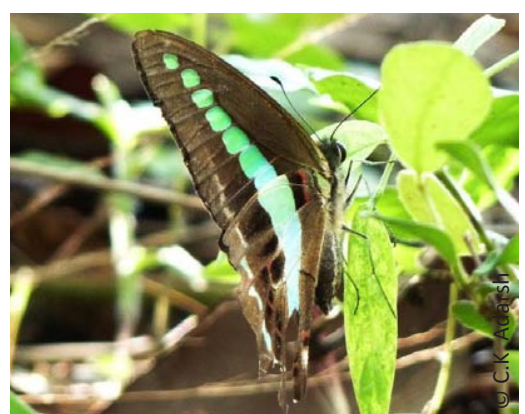

Image 3. Graphium sarpedon Common Bluebottle

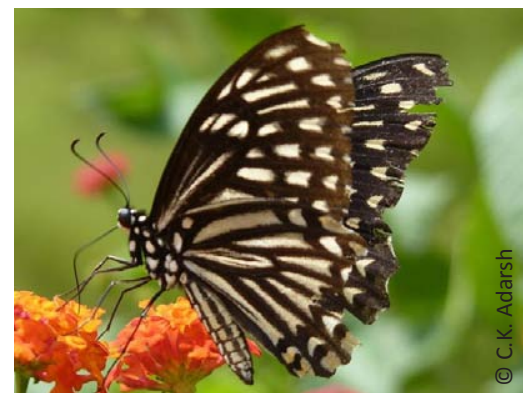

Image 6. Papilio clytia Common Mime

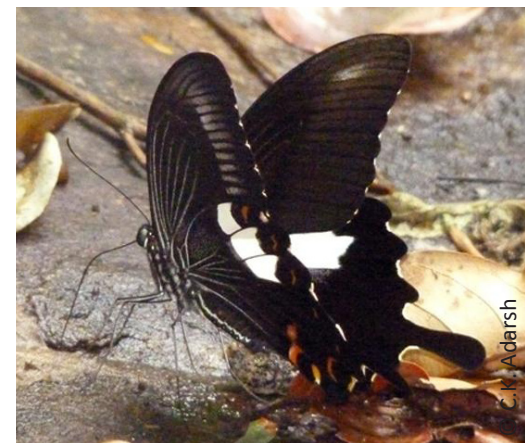

Image 9. Papilio helenus Red Helen

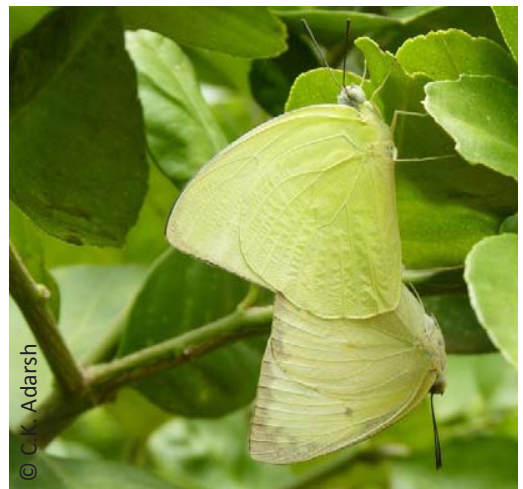

Image 12. Catopsilia pomona Common Emigrant 


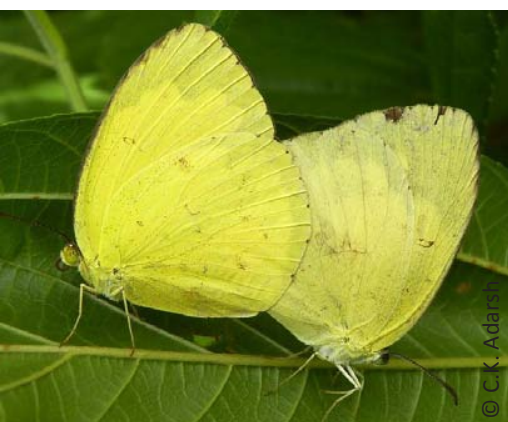

Image 13. Eurema hecabe Common Grass Yellow

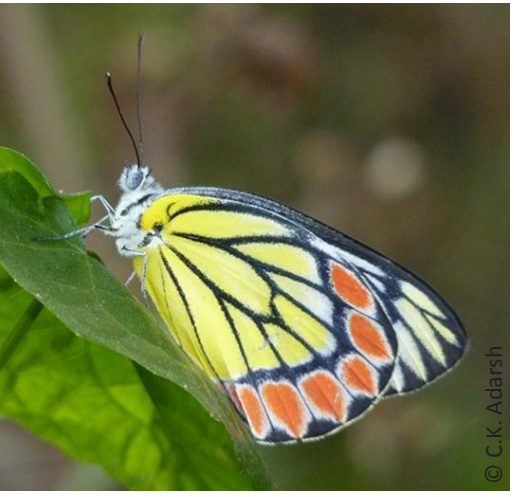

Image 16. Delias eucharis Common Jezebel

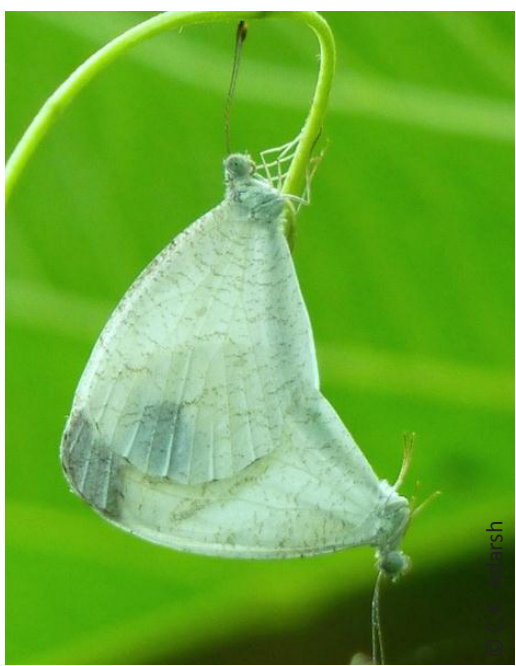

Image 19. Leptosia nina Psyche

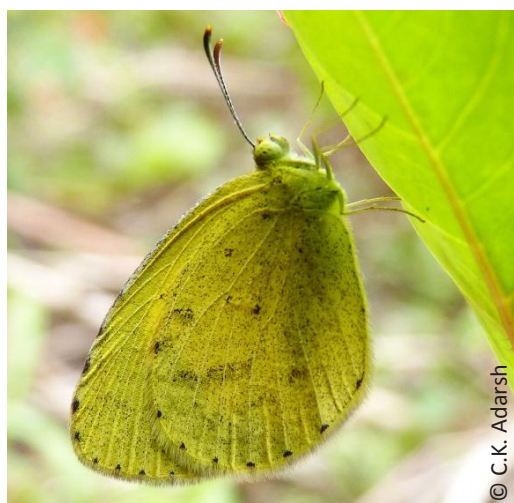

Image 14. Eurema brigitta Small grass yellow

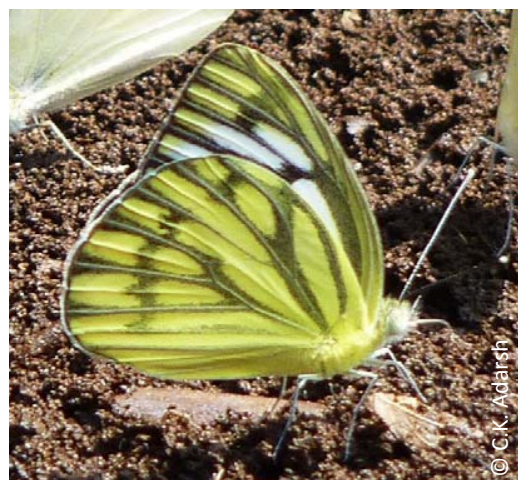

Image 17. Cepora nerissa Common gull

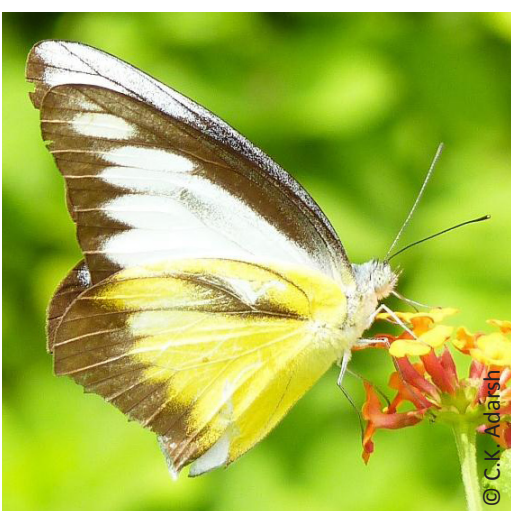

Image 20. Appias Iyncida Chocolate Albatross

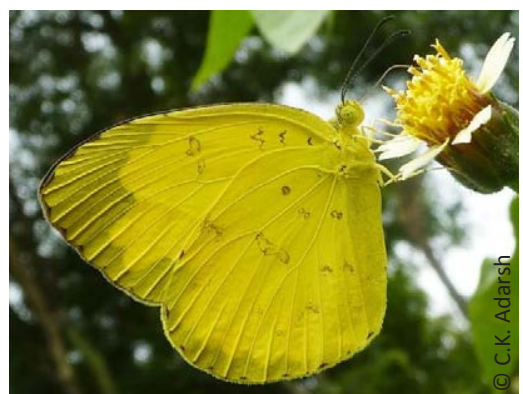

Image 15. Eurema blanda Three-spot Grass Yellow

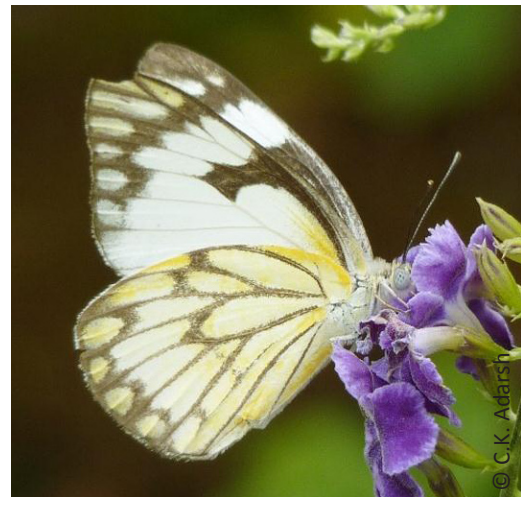

Image 18. Belenois aurota Pioneer

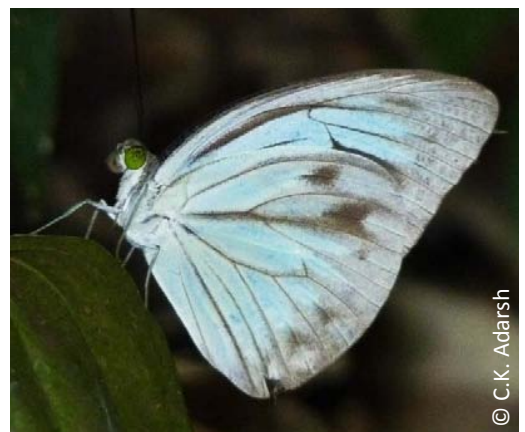

Image 21. Pareronia hippie Common Wanderer

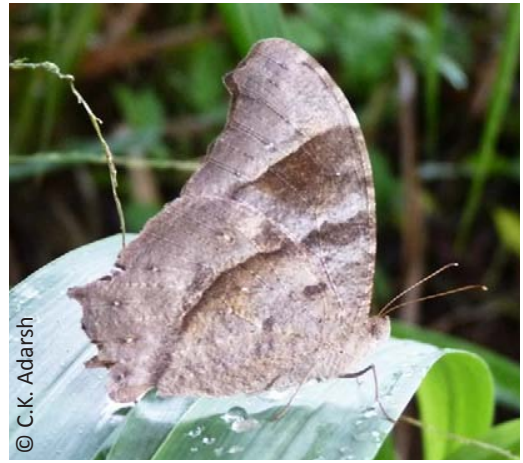

Image 22. Melanitis leda Common Evening Brown 


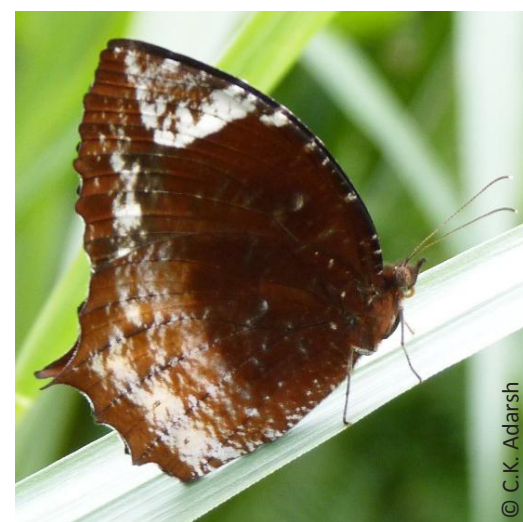

Image 23. Elymnias hypermnestra Common Palmfly

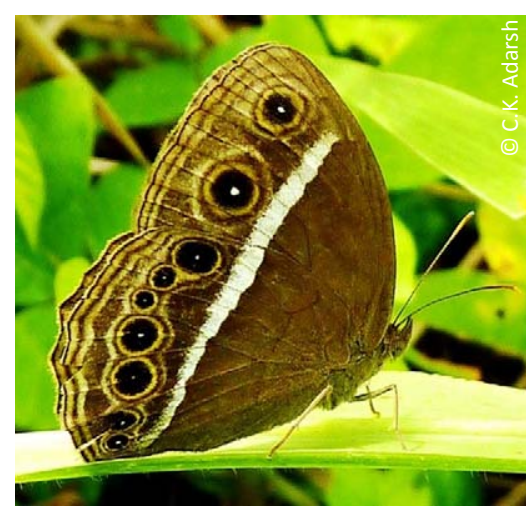

Image 26. Mycalesis perseus Common Bushbrown

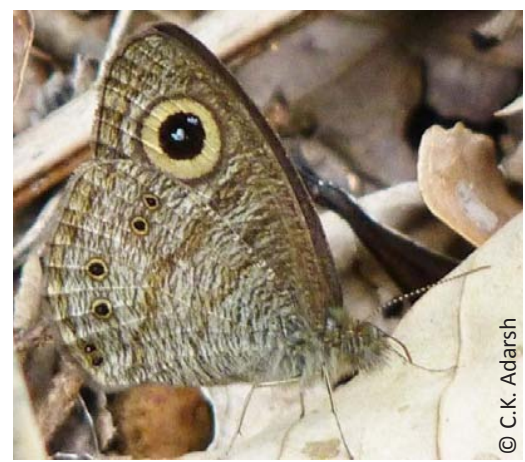

Image 29. Ypthima baldus Common Fivering

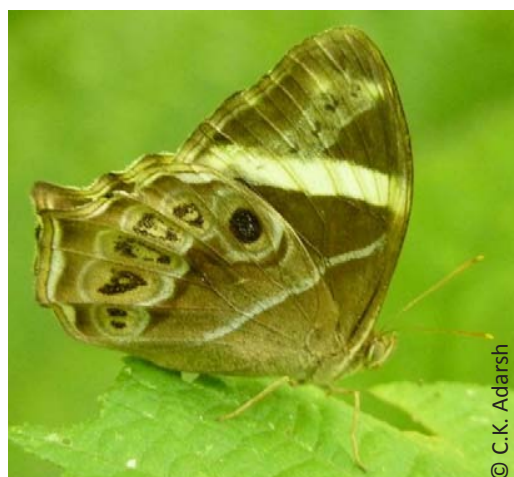

Image 24. Lethe europa Bamboo Tree brown

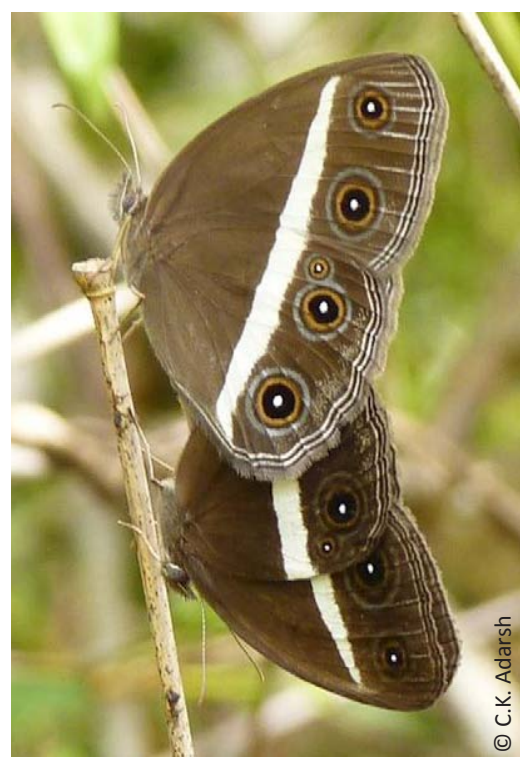

Image 27. Orsotriaena medus Nigger

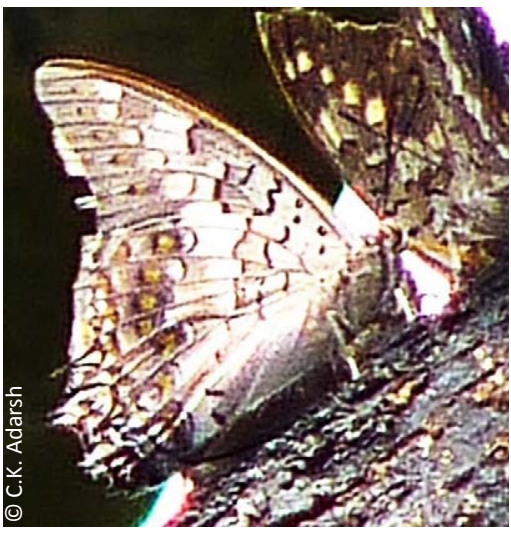

Image 30. Charaxes solon Black Rajah

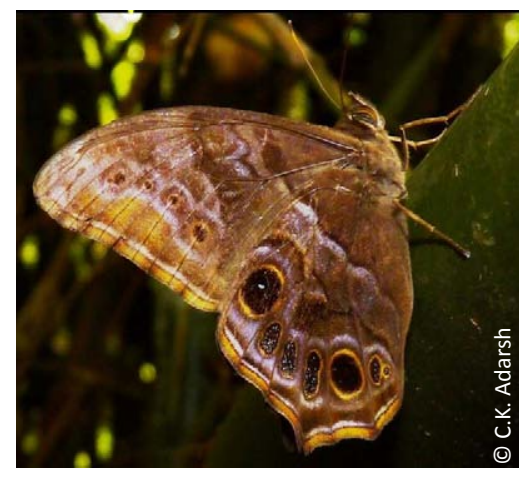

Image 25. Lethe drypetis Tamil Tree Brown

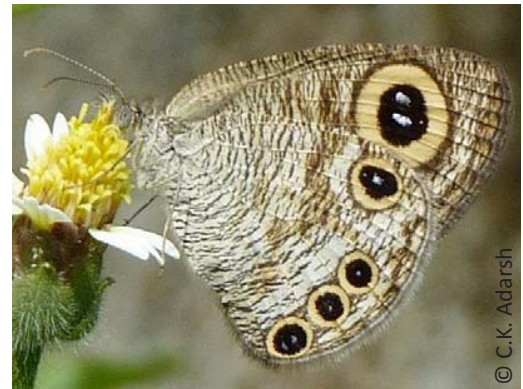

Image 28. Ypthima huebneri Common Fourring

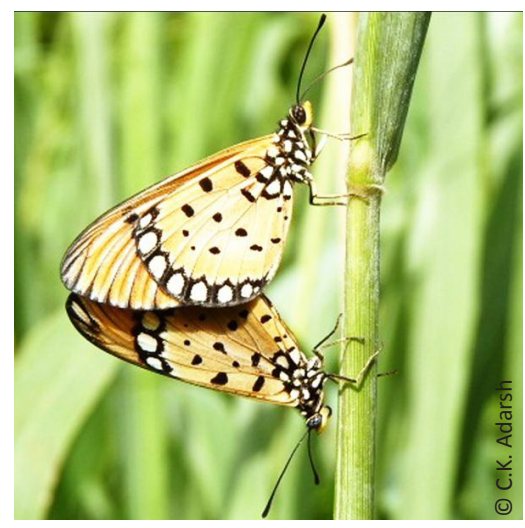

Image 31. Acraea violae Tawny Coster

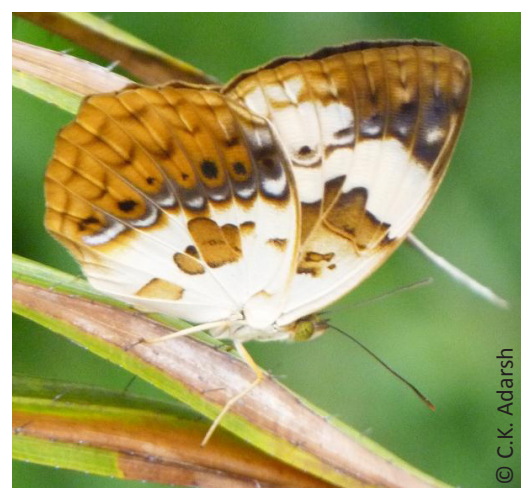

Image 32. Cupha erymanthis Rustic 


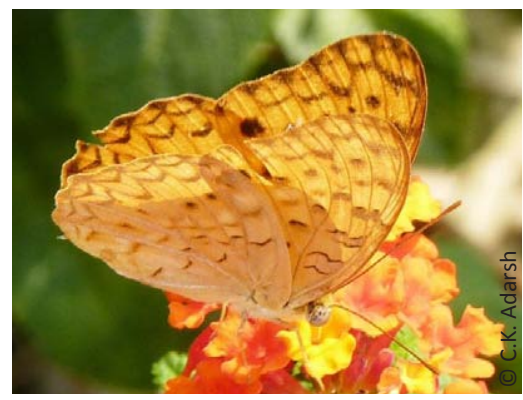

Image 33. Phalanta phalantha Common Leopard

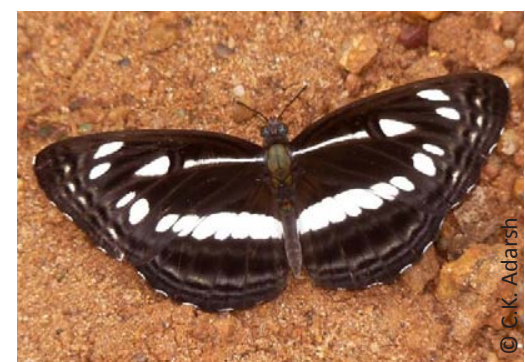

Image 36. Neptis jumbah Chestnut-streaked Sailer

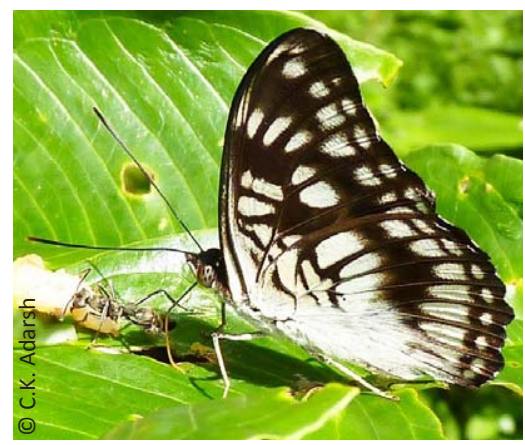

Image 39. Athyma ranga Blackvein Sergeant

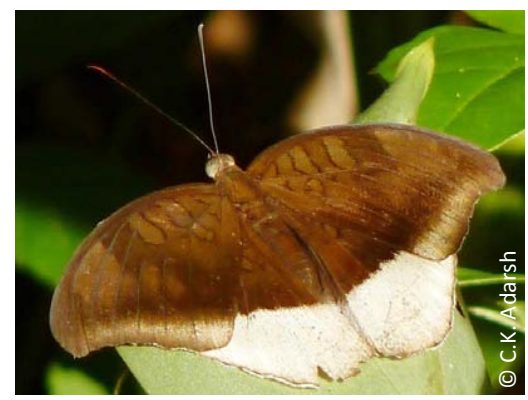

Image 42. Tanaecia lepidea Grey Count

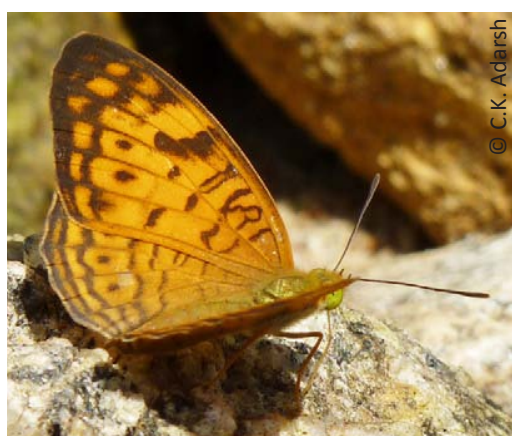

Image 34. Phalanta alcippe mercea Small leopard

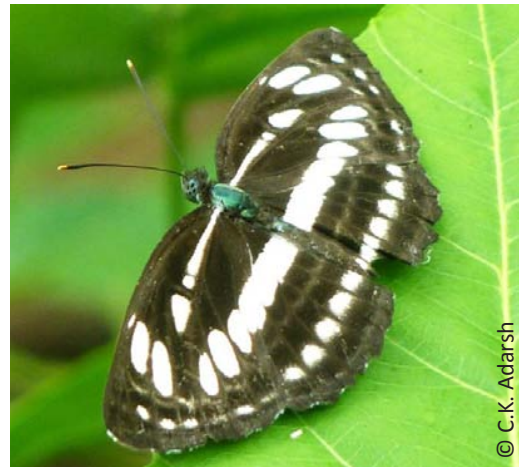

Image 37. Neptis hylas Common Sailer

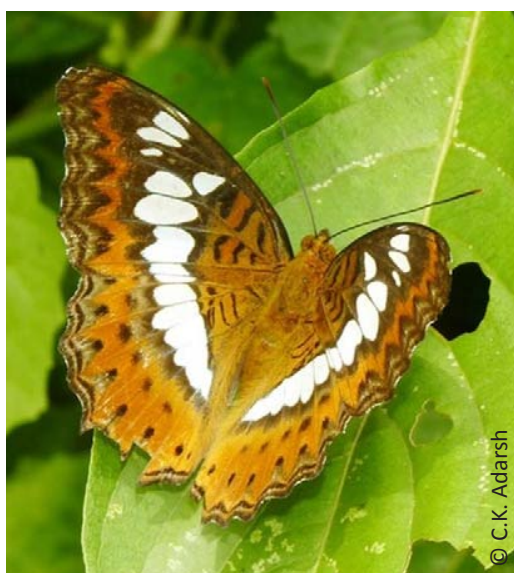

Image 40. Limenitis procris Commander

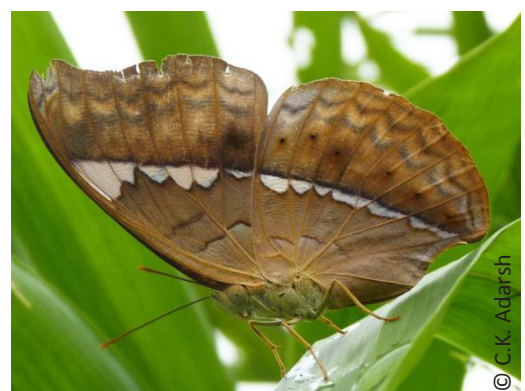

Image 35. Cirrochroa thais Tamil Yeoman

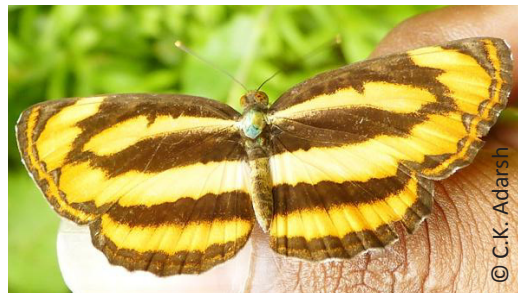

Image 38. Pantoporia hordonia Common Lascar

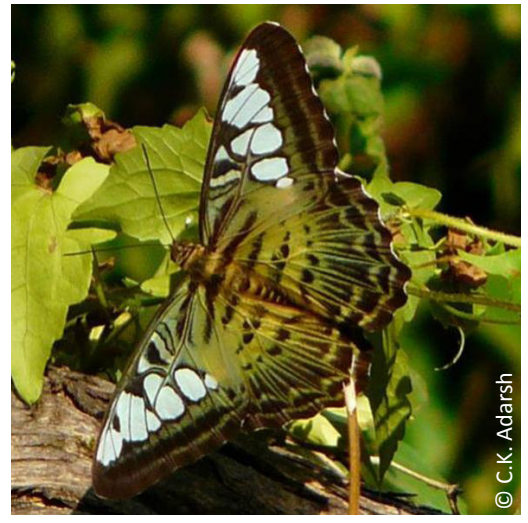

Image 41. Parthenos sylvia Clipper

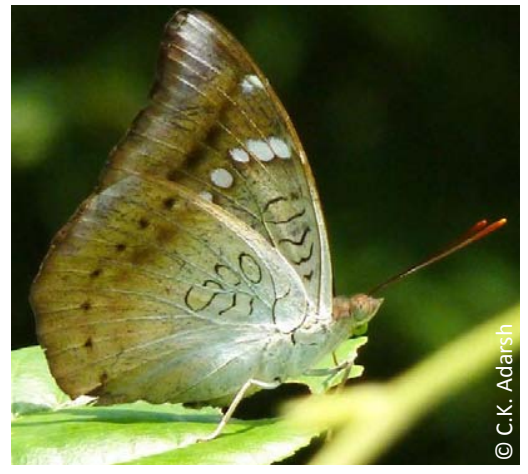

Image 43. Euthalia aconthea Common Baron 


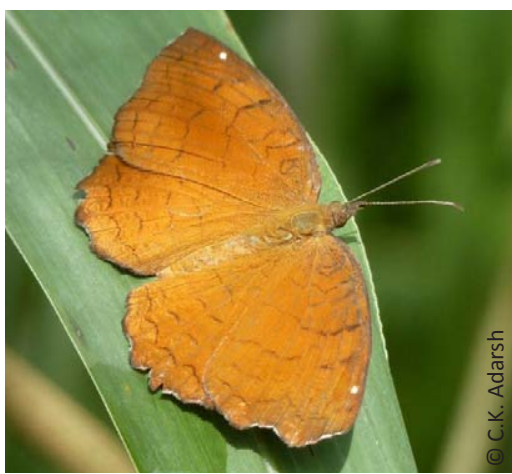

Image 44. Ariadne ariadne Angled Castor

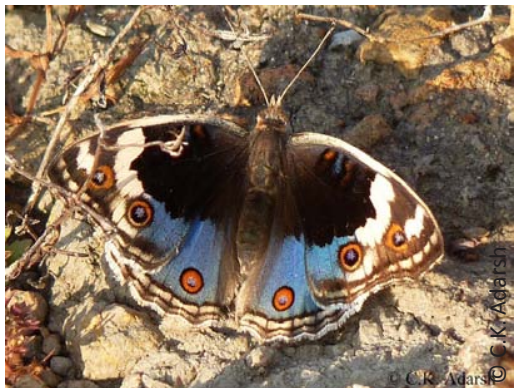

Image 47. Junonia orithya Blue Pansy

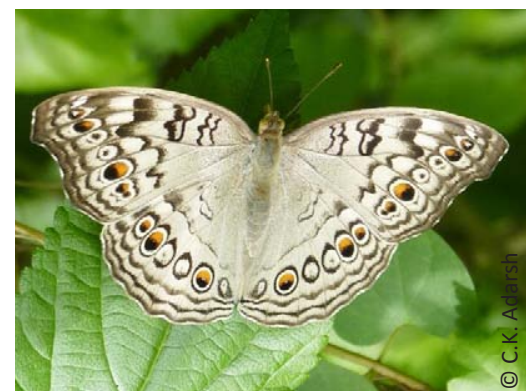

Image 50. Junonia atlites Grey Pansy

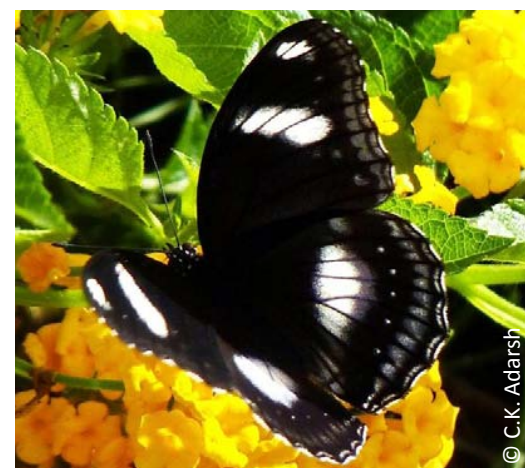

Image 53. Hypolimnas bolina Great Eggfly

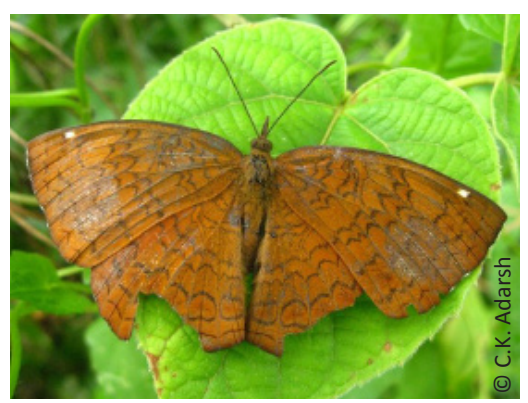

Image 45. Ariadne merione Common Castor

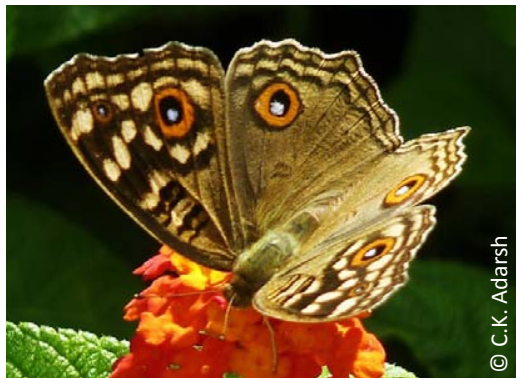

Image 48. Junonia lemonias Lemon Pansy

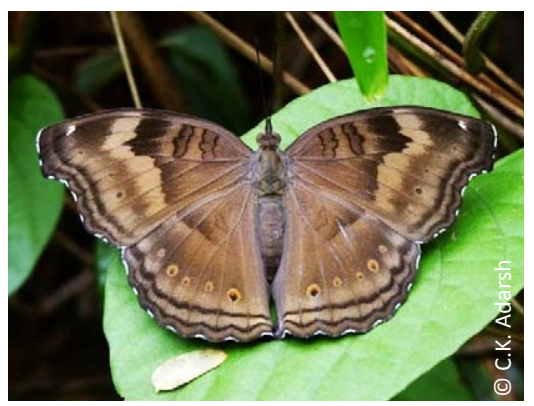

Image 51. Junonia iphita Chocolate Pansy

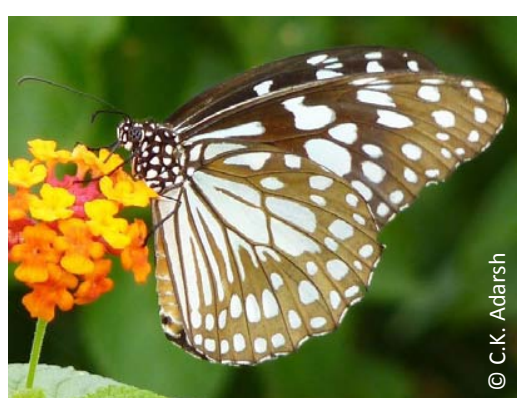

Image 54. Tirumala limniace-Blue Tiger

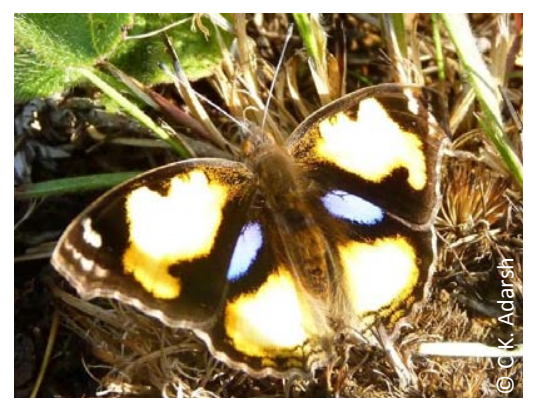

Image 46. Junonia hierta Yellow Pansy

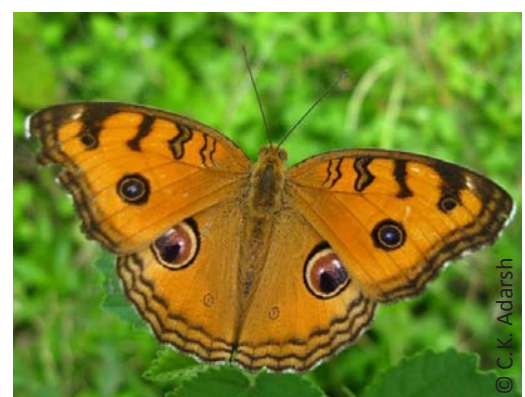

Image 49. Junonia almanac Peacock Pansy

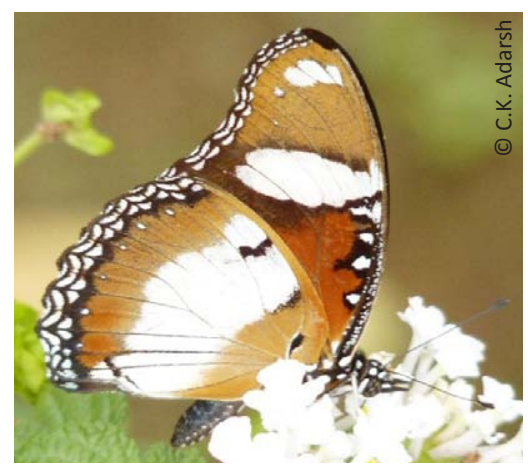

Image 52. Hypolimnas misippus Danaid Eggfly

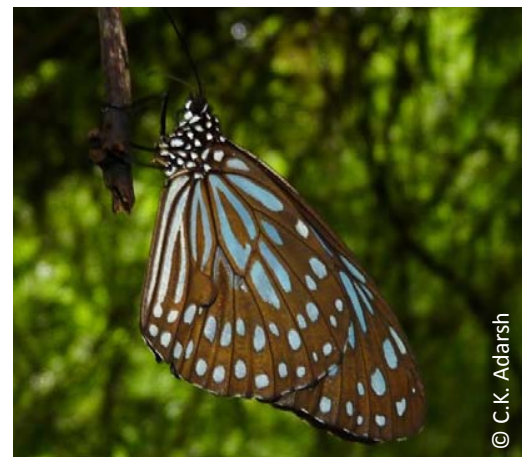

Image 55. Tirumala septentrionis Dark Blue Tiger 


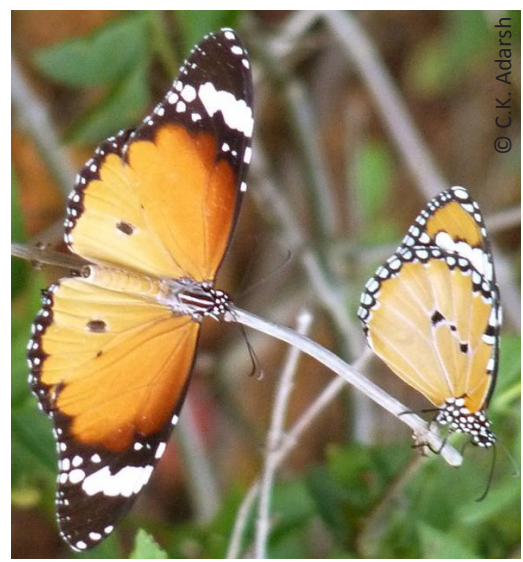

Image 56. Danaus chrysippus Plain Tiger

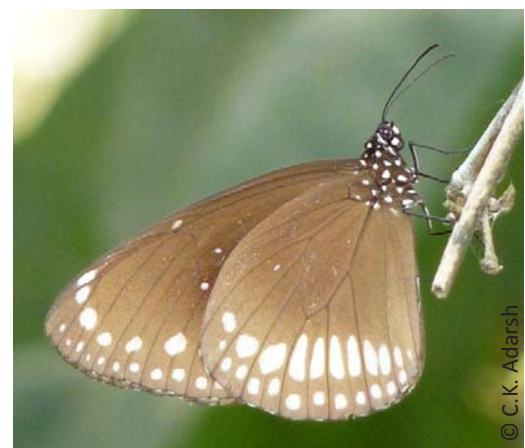

Image 59. Euploea klugii Brown King Crow

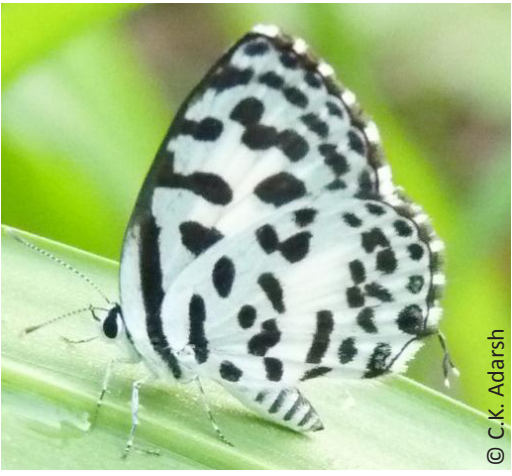

Image 62. Castalius rosimon Common Pierrot

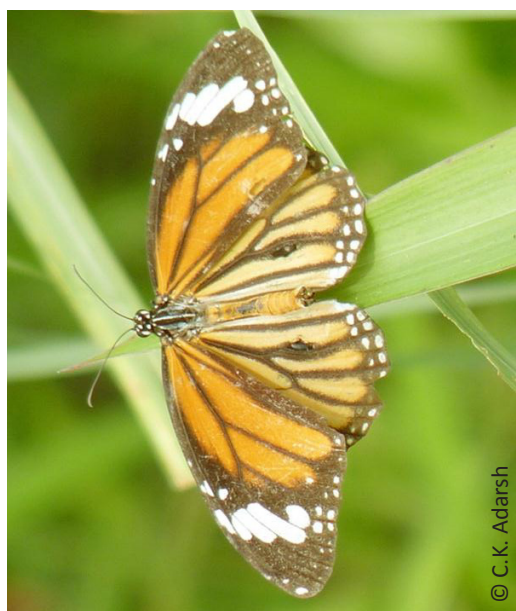

Image 57. Danaus genutia Striped Tiger

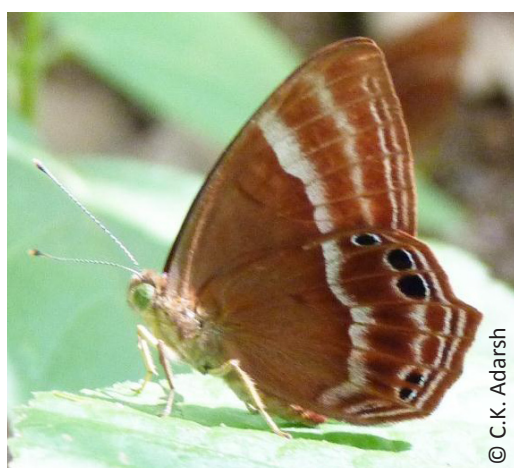

Image 60. Abisera echerius Plum Judy

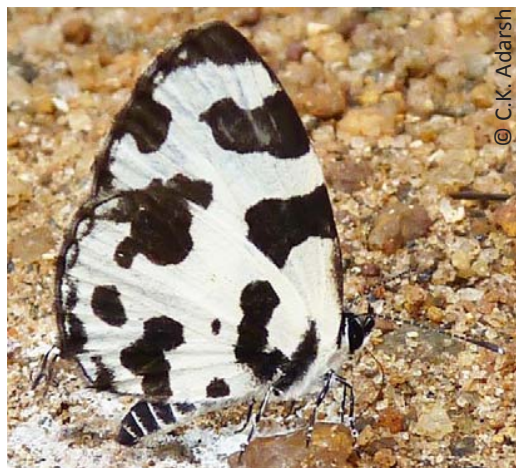

Image 63. Caleta caleta Angled Pierrot

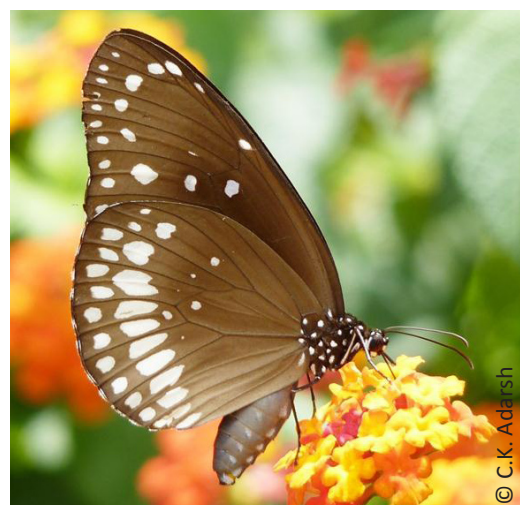

Image 58. Euploea core Common Crow

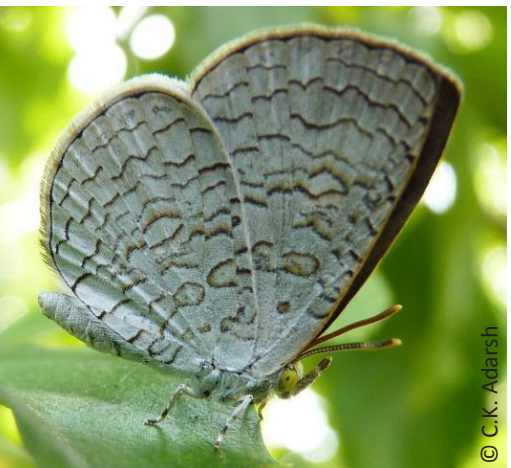

Image 61. Spalgis epius Apefly

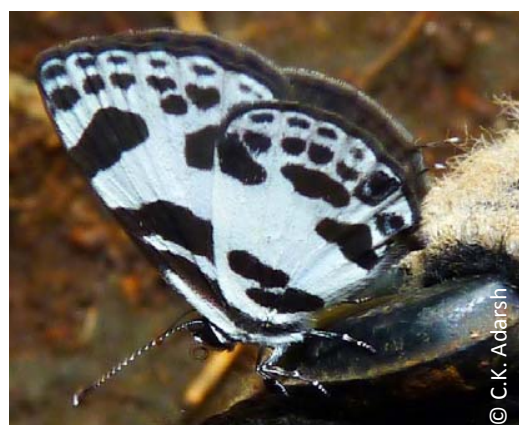

Image 64. Discolampa ethion Banded Blue Pierrot

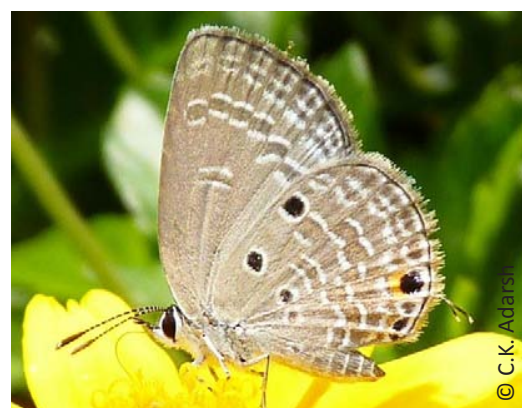

Image 65. Chilades pandava Plains Cupid 


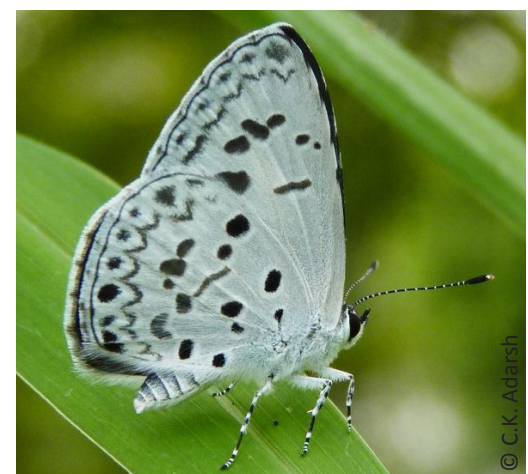

Image 66. Acytolepis puspa Common Hedge Blue.

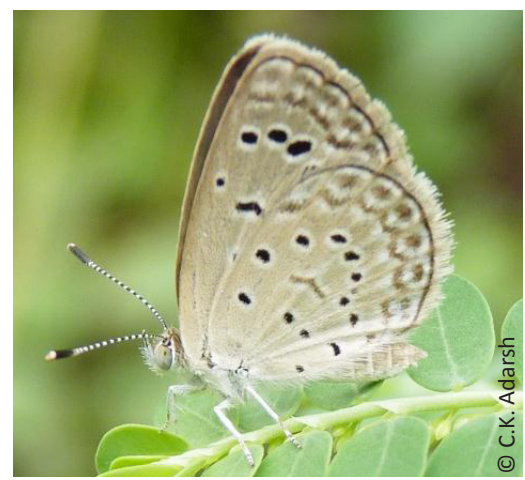

Image 69. Zizula hylax Tiny Grass Blue.

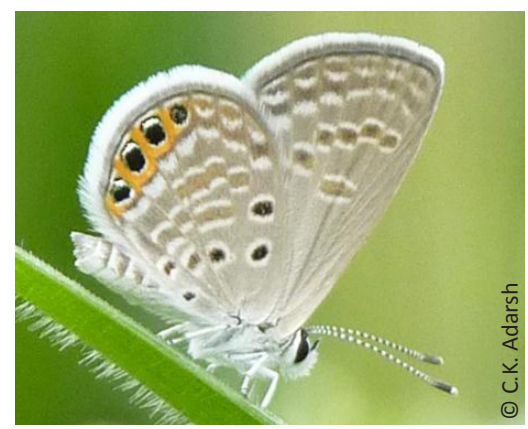

Image 72. Freyeria putli Grass Jewel

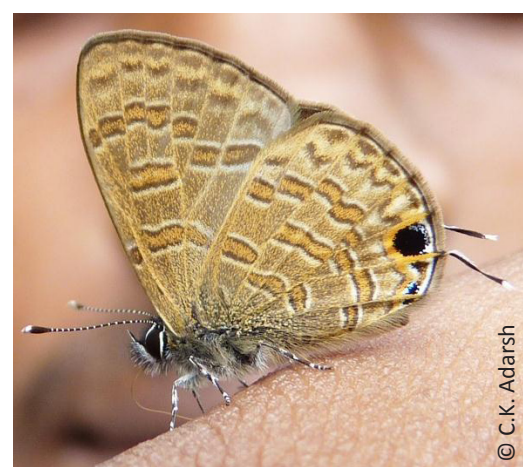

Image 75. Prosotas nora Common Line-blue

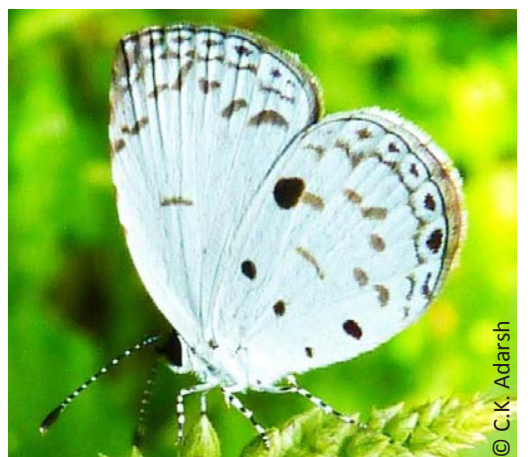

Image 67. Neopithecops zalmora Quaker

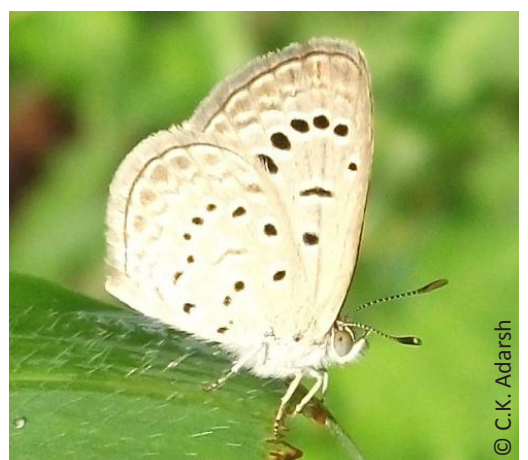

Image 70. Pseudozizeeria maha Pale grass blue

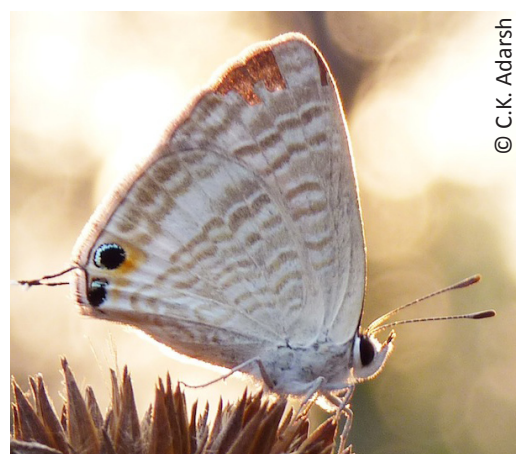

Image 73. Lampides boeticus Pea Blue

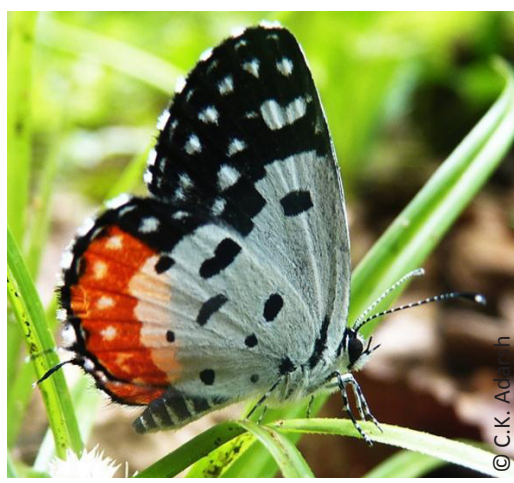

Image 76. Talicada nyseus Red Pierrot

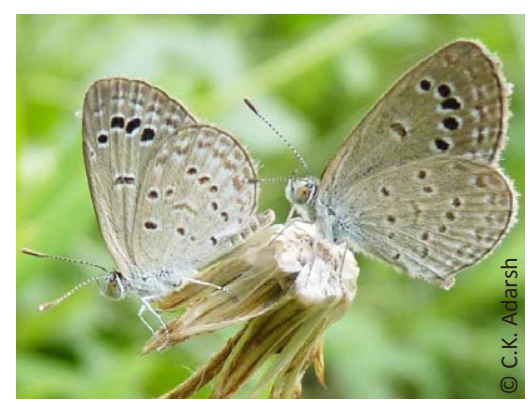

Image 68. Zizina otis Lesser Grass Blue

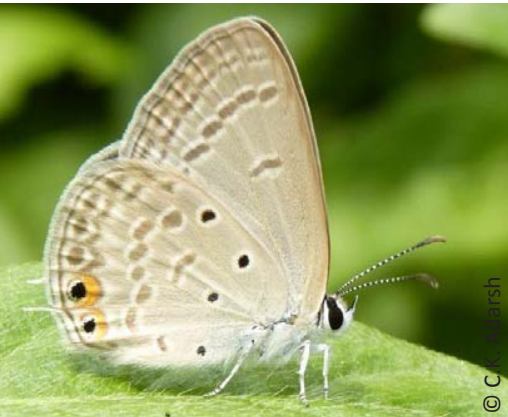

Image 71. Euchrysopsc nejus Gram Blue.

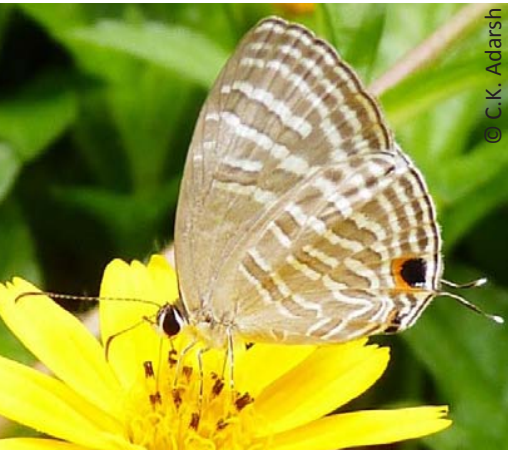

Image 74. Jamides celeno Common Cerulean

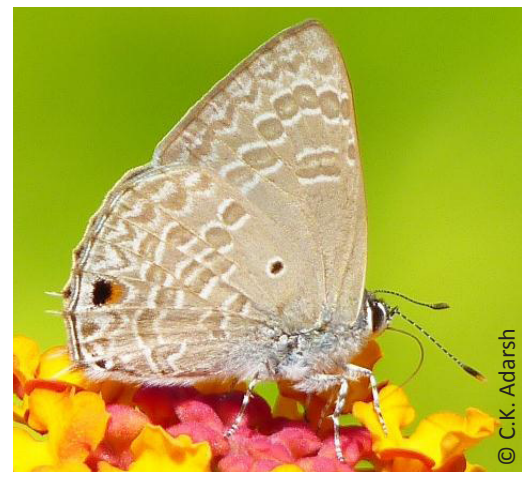

Image 77. Anthene lycaenina Pointed Ciliate Blue 


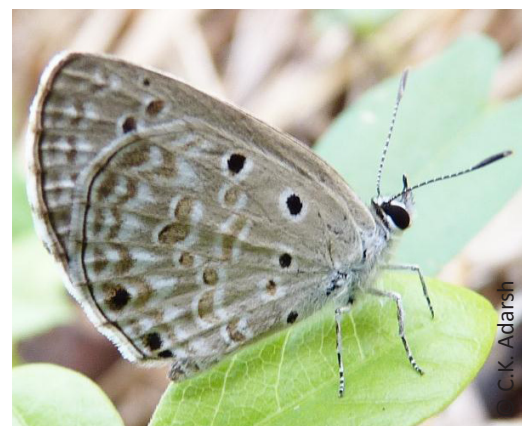

Image 78. Chilades Iajus Lime Blue

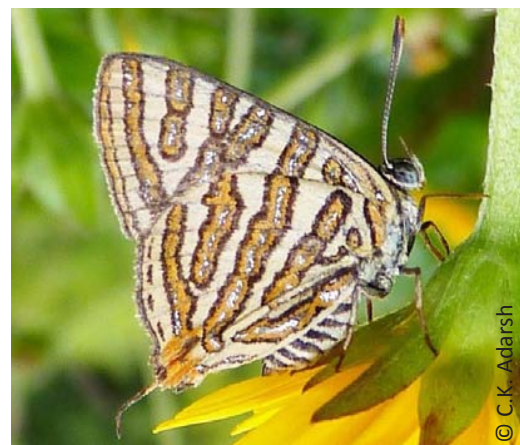

Image 81. Spindasis vulcanus Common Silverline

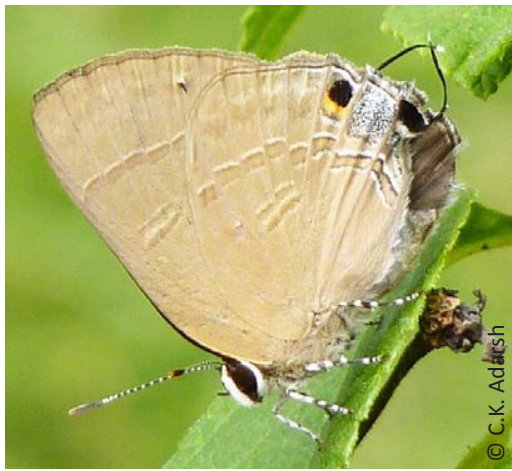

Image 84. Rapala manea Slate Flash

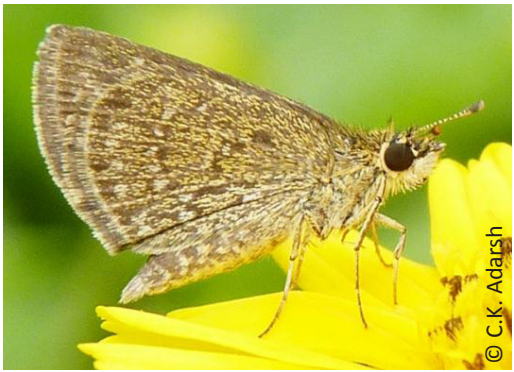

Image 87. Aeromachus pygmaeus Pygmyscrub Hopper

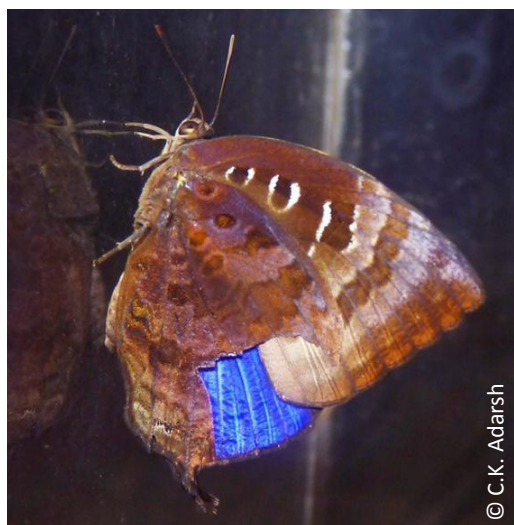

Image 79. Arhopala pseudocentaurus Western Centaur Oakblue

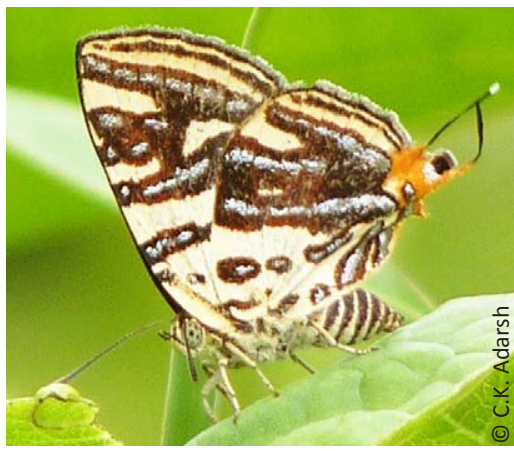

Image 82. Spindasis elima Scarce Silverline

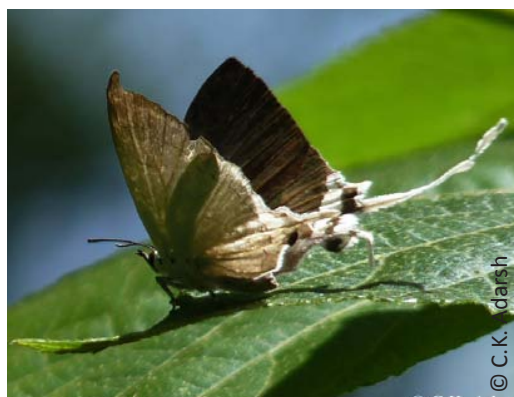

Image 85. Cheritra freja Common Imperial

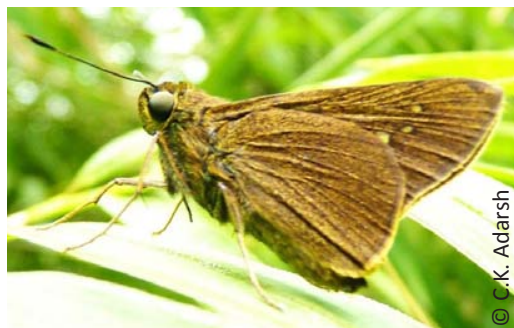

Image 88. Thoressa stigmata Unbranded Ace

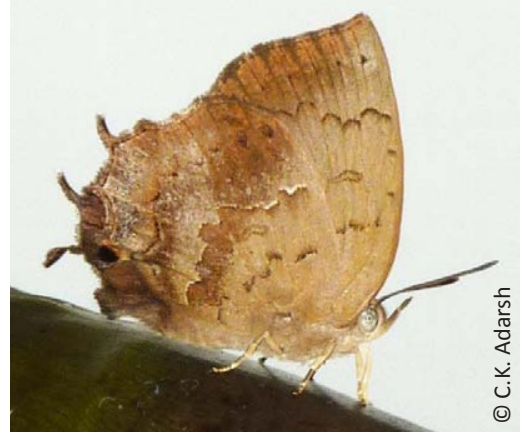

Image 80. Surendra querecetorum Common Acacia Blue

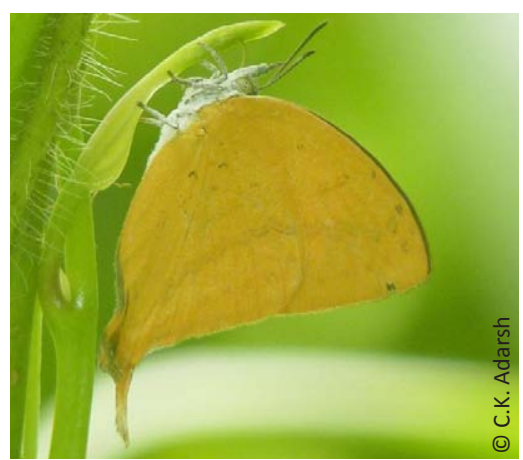

Image 83. Loxura atymnus Yamfly

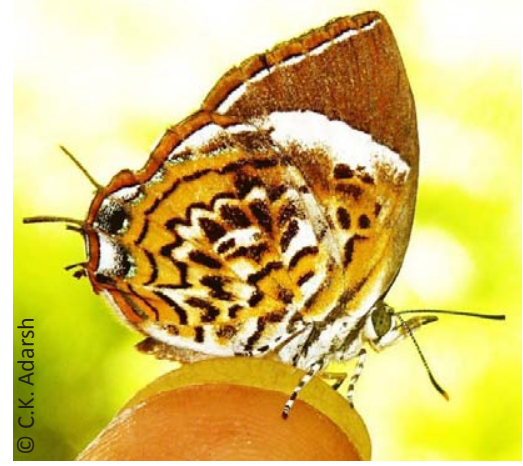

Image 86. Rathinda amor Monkey Puzzle

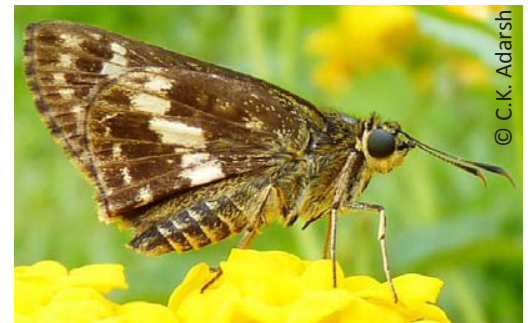

Image 89. Halpe porus Moore's Ace 


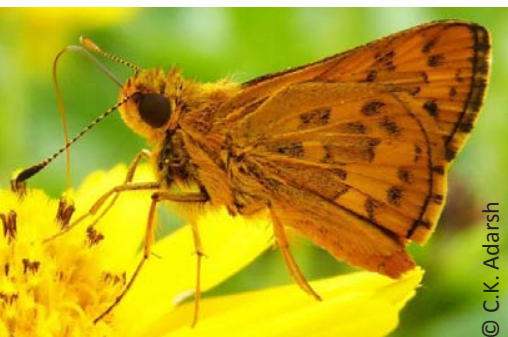

Image 90. Ampittia dioscorides Bush Hopper

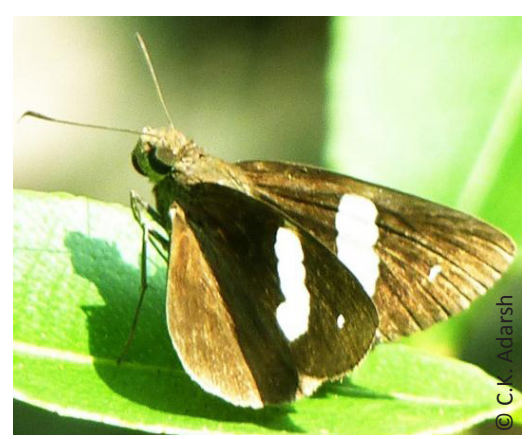

Image 93. Notocrypta paralysos Common Banded Demon

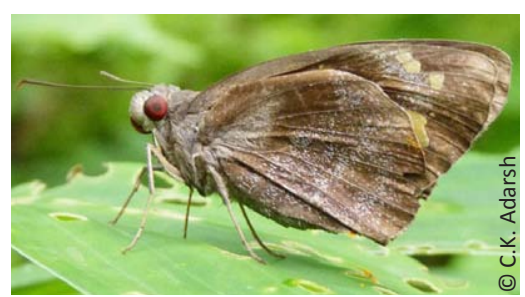

Image 96. Gangara thyrsis Giant Red-eye

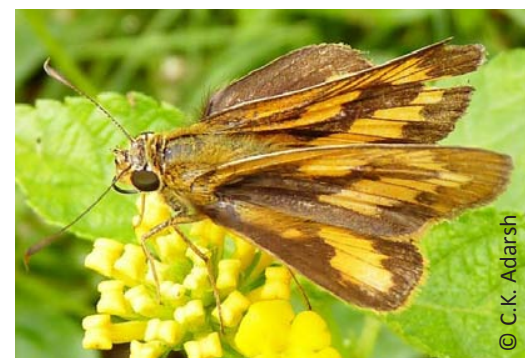

Image 99. Telicota colon Pale Palm Dart

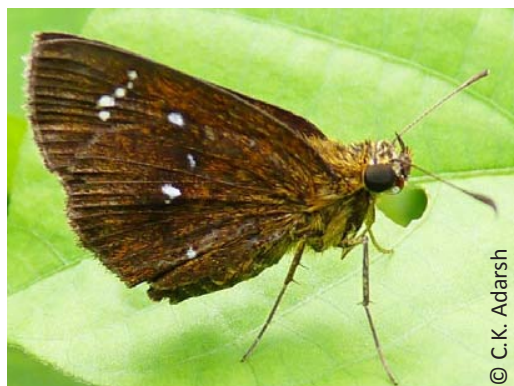

Image 91. Iambrix salsala Chestnut Bob

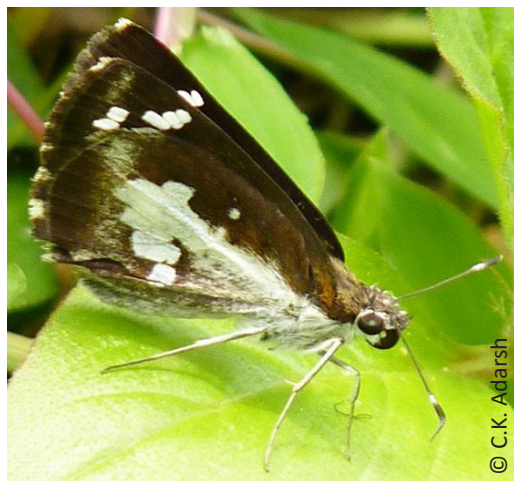

Image 94. Udaspes folus Grass Demon.

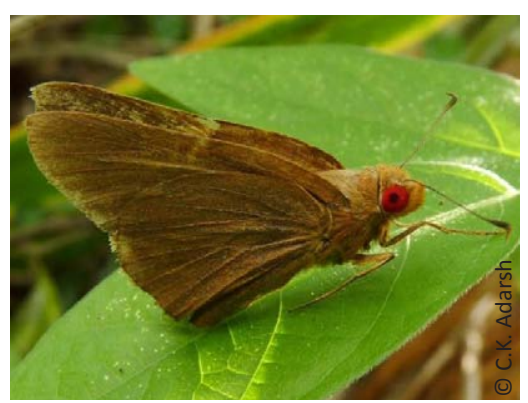

Image 97. Matapa aria Common Red-eye

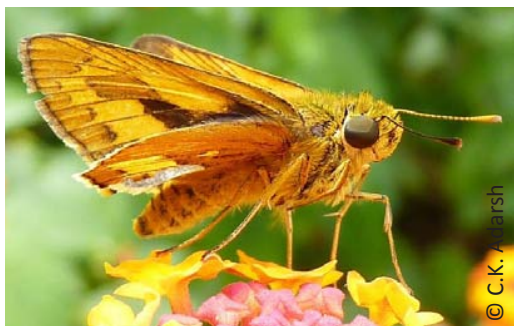

Image 100. Telicota ancilla Dark Palm Dart

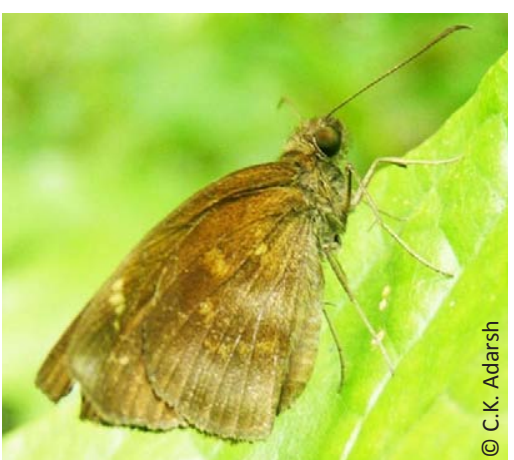

Image 92. Psolos fuligo Coon

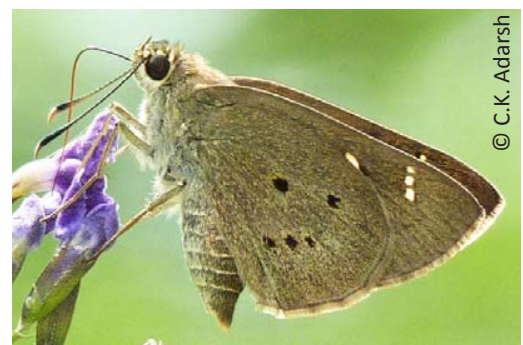

Image 95. Suastus gremius Indian Palm Bob

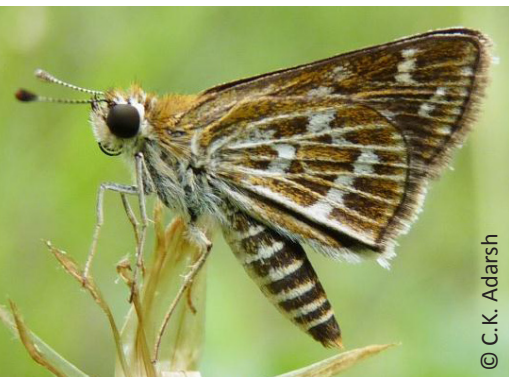

Image 98. Taractro ceramaevius Common Grass Dart

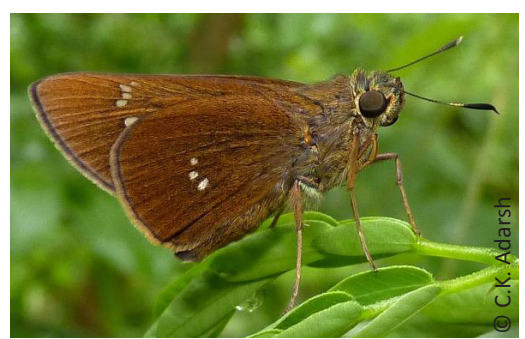

Image 101. Borbo bevani Bevan's Swift 


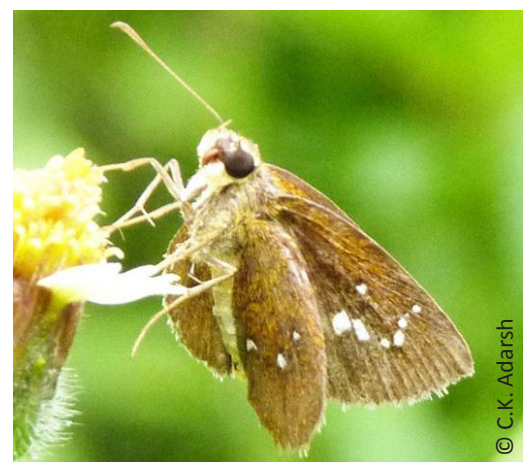

Image 102. Borbo cinnara Rice Swift

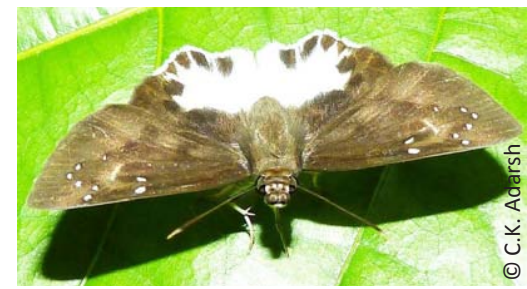

Image 105. Tagiades litigiosa Water Snow Flat

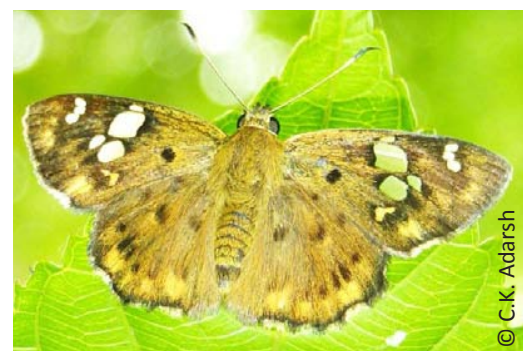

Image 108. Coladenia indrani Tricoloured Pied Flat

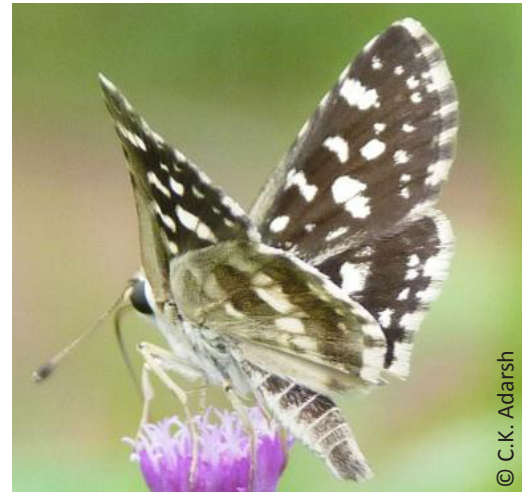

Image 111. Spialia galba Indian Grizzled Skipper

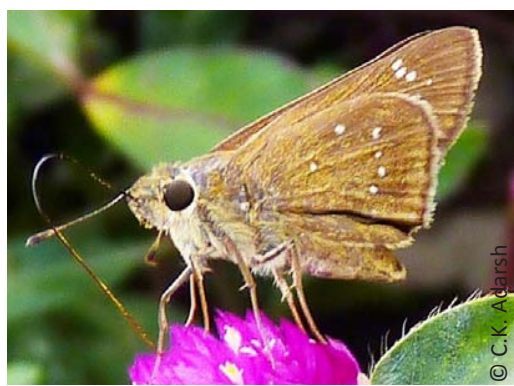

Image 103. Pelopidas mathias Small Branded Swift

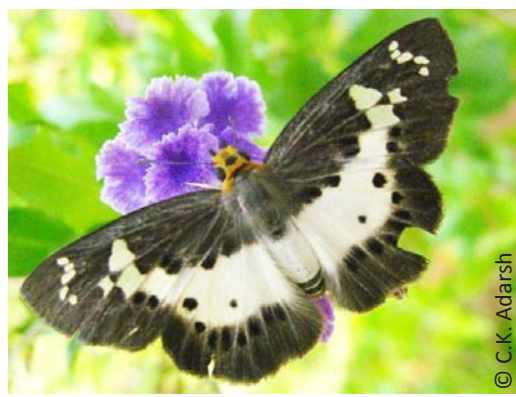

Image 106. Gerosis bhagava Common Yellow-breasted Flat

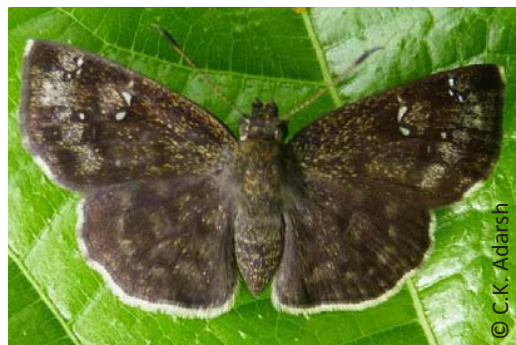

Image 109. Sarangesa dasahara Common Small Flat

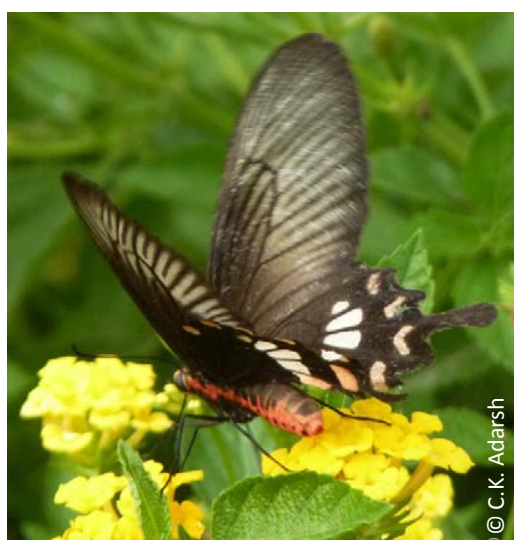

Image 112. Pachliopta aristolochiae aristolochiae Common Rose

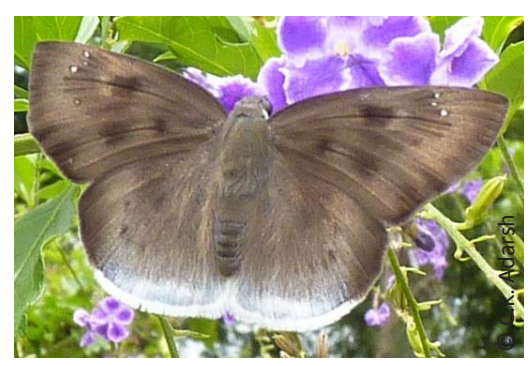

Image 104. Tagiades gana Suffused Snow Flat

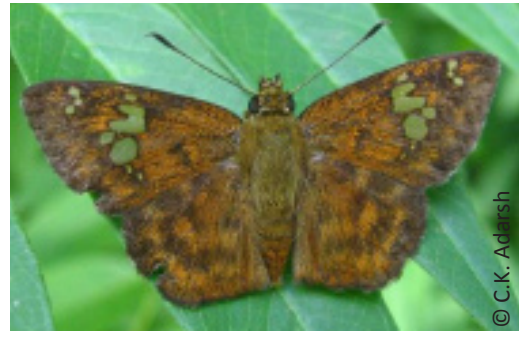

Image 107. Pseudocoladenia dan Fulvous Pied Flat

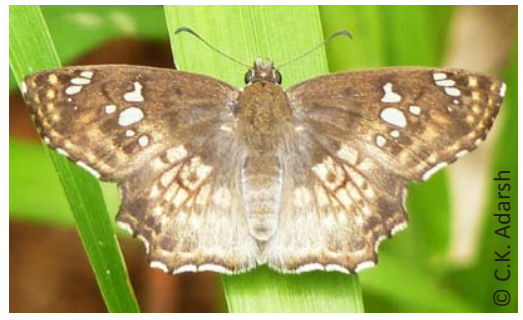

Image 110. Odontoptilum ransonnetii Golden Angle

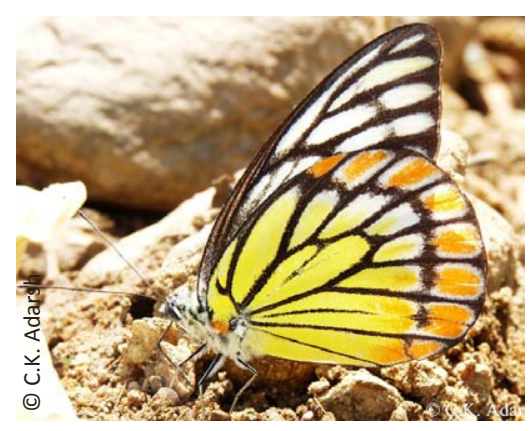

Image 113. Prioneris sita Painted Sawtooth 


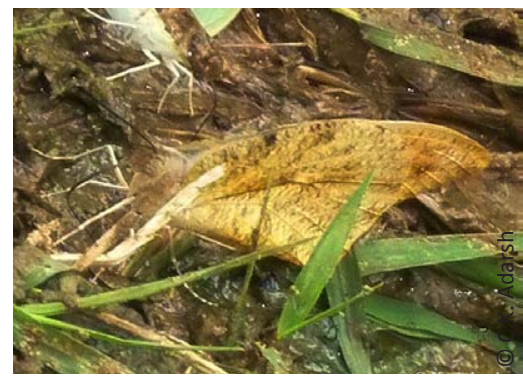

Image 114. Hebemoia glaucippe australis Great Orange Tip

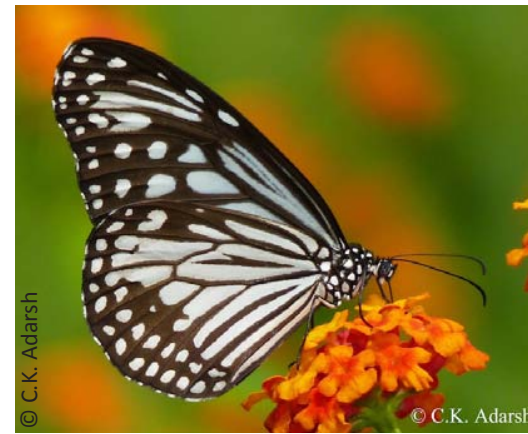

Image 117. Parantica aglea aglea Glassy Blue Tiger

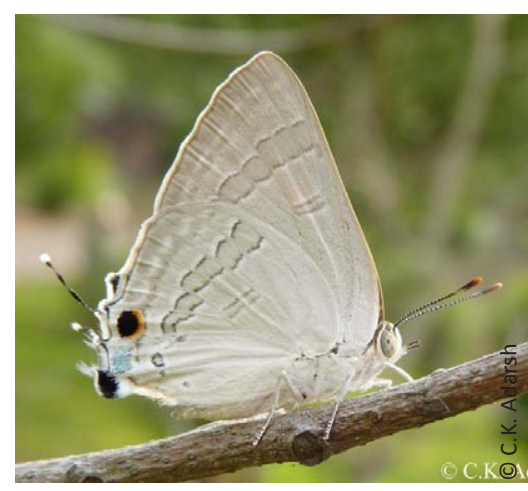

Image 120. Virachola isocrates Common Guava Blue

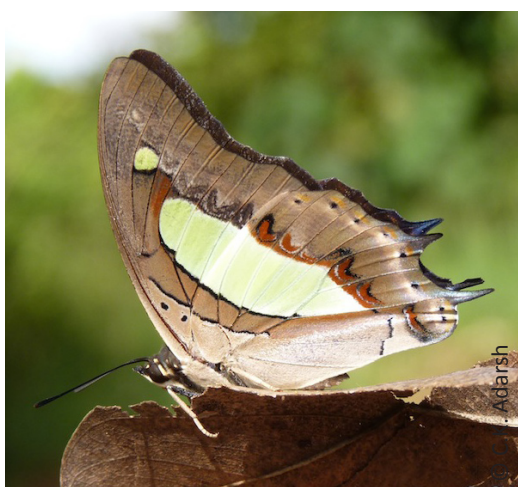

Image 115. Charaxes athamas athamas Common Nawab

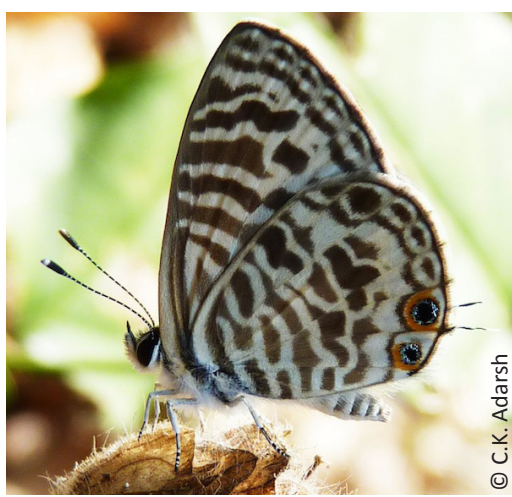

Image 118. Leptotes plinius plinius Zebra Blue

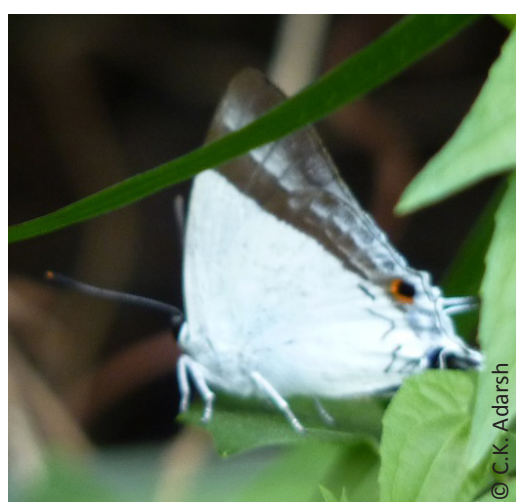

Image 121. Rachana jalindra macanita Banded Royal

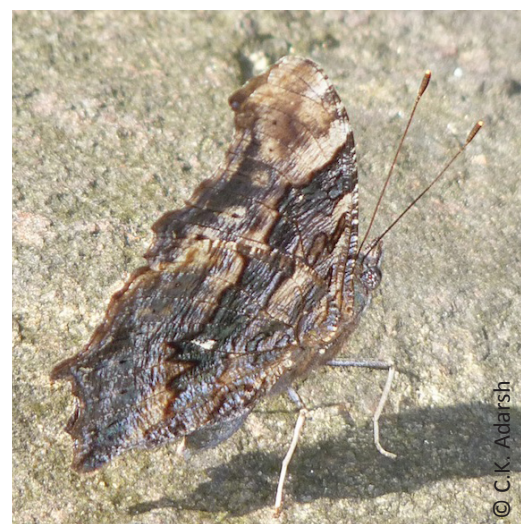

Image 116. Kaniska canace viridis Blue Admiral

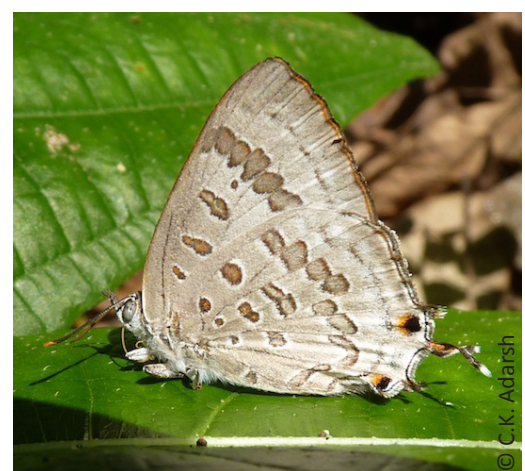

Image 119. Zesius chrysomallus Red Spot

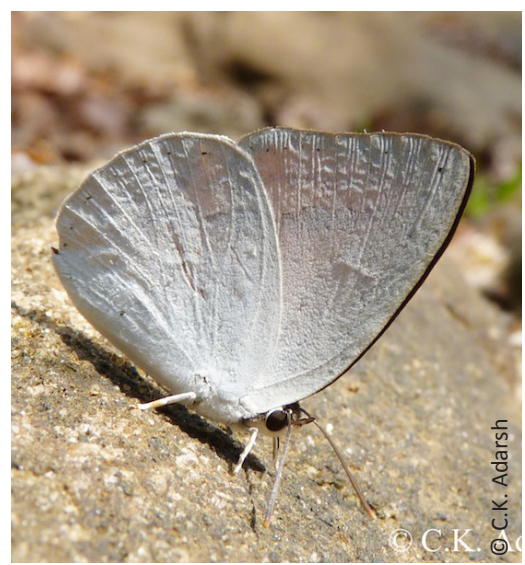

Image 122. Curetis thetis Indian Sunbeam 


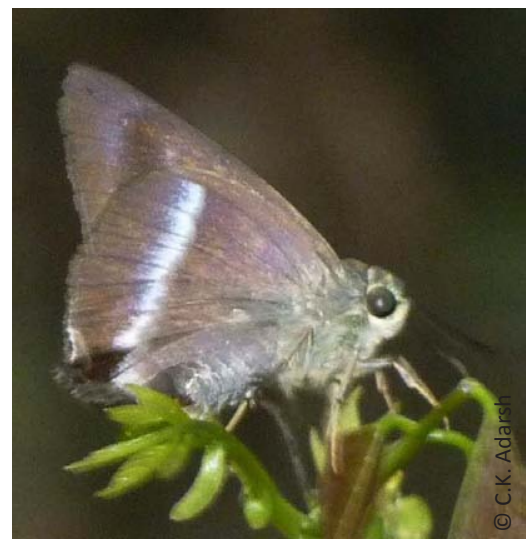

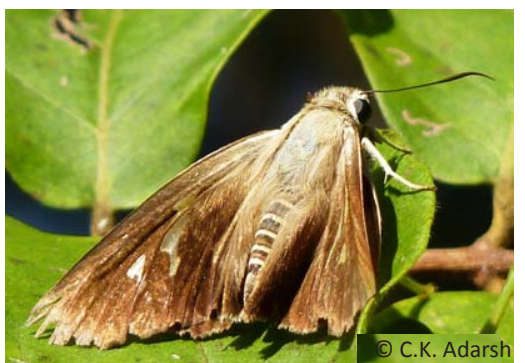

Image 124. Badamia exclamationis Brown Awl

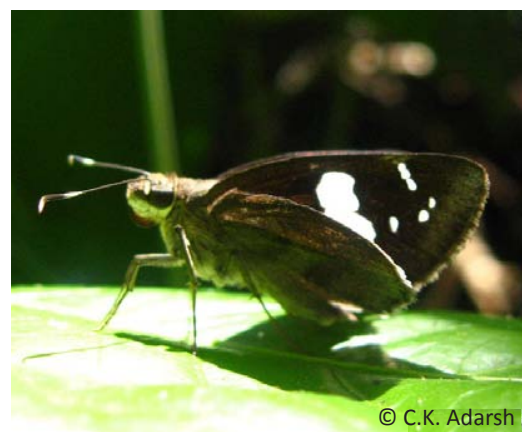

Image 125. Notocrypta curvifascia curvifascia Restricted Demon

Image 123. Hasora chromus chromus

Common Banded Awl 